Supporting Information for:

\title{
Aromatic Esters of the Crinane Amaryllidaceae Alkaloid Ambelline as Selective Inhibitors of Butyrylcholinesterase
}

Jana Mařiková, ${ }^{\dagger}$ Aneta Ritomská, ${ }^{\ddagger}$ Jan Korábečný,,\|\| Rozálie Peřinová, ${ }^{\ddagger}$ Abdullah Al Mamun, ${ }^{\ddagger}$ Tomáš Kučera, ${ }^{\nabla}$ Eliška Kohelová, ${ }^{\ddagger}$ Daniela Hulcová, ${ }^{\ddagger}, \perp$ Tereza Kobrlová, ${ }^{\nabla, \|}$ Jiři Kuneš, ${ }^{\dagger}$ Lucie Nováková, $\$$ Lucie Cahlíková*, $\neq$

tDepartment of Bioorganic and Organic Chemistry, ‡ADINACO Research Group, Department of Pharmaceutical Botany, ${ }^{\perp}$ Department of Pharmacognosy and \$Department of Analytical Chemistry, Faculty of Pharmacy, Charles University, Heyrovskeho 1203, 50005 Hradec Kralove, Czech Republic

${ }^{\nabla}$ Department of Toxicology and Military Pharmacy, Faculty of Military Health Sciences, University of Defence, Trebesska 1575, 50001 Hradec Kralove, Czech Republic.

"Biomedical Research Centre, University Hospital Hradec Kralove, Sokolska 581, 500 05 Hradec Kralove, Czech Republic 


\section{Table of contents}

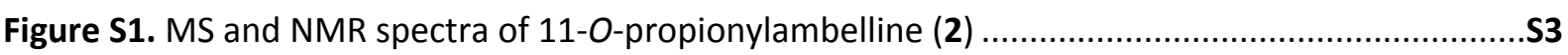

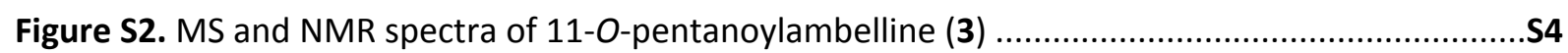

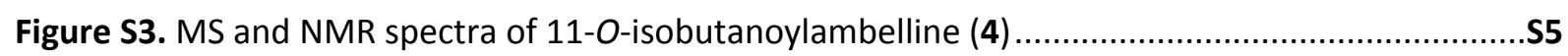

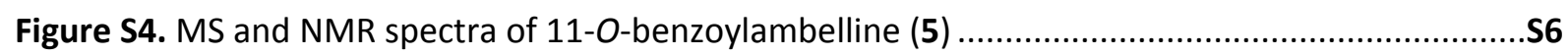

Figure S5. MS and NMR spectra of 11-O-(2-methylbenzoyl)ambelline (6) .......................................S7

Figure S6. MS and NMR spectra of 11-O-(3-methylbenzoyl)ambelline (7) .......................................S8

Figure S7. MS and NMR spectra of 11-O-(4-methylbenzoyl)ambelline (8) ......................................S9

Figure S8. MS and NMR spectra of 11-O-(2-methoxybenzoyl)ambelline (9) ....................................S10

Figure S9. MS and NMR spectra of 11-O-(3-methoxybenzoyl)ambelline (10) .................................S11

Figure S10. MS and NMR spectra of 11-O-(4-methoxybenzoyl)ambelline (11) ................................S12

Figure S11. MS and NMR spectra of 11-O-(2-nitrobenzoyl)ambelline (12) .....................................S13

Figure S12. MS and NMR spectra of 11-O-(3-nitrobenzoyl)ambelline (13) .....................................S14

Figure S13. MS and NMR spectra of 11-O-(4-nitrobenzoyl)ambelline (14) .....................................S15

Figure S14. MS and NMR spectra of 11-O-(2-naphthoyl)ambelline (15).........................................S16

Figure S15. MS and NMR spectra of 11-O-(1-naphthoyl)ambelline (16)..........................................S17

Figure S16. MS and NMR spectra of 11-O-(2-furoyl)ambelline (17)...............................................S18

Figure S17. MS and NMR spectra of 11-O-(3-furoyl)ambelline (18)..............................................S19

Figure S18. MS and NMR spectra of 11-O-(3,4-dimethoxybenzoyl)ambelline (19) ...........................S20

Figure S19. MS and NMR spectra of 11-O-(3,5-diethoxybenzoyl)ambelline (20)..............................S21

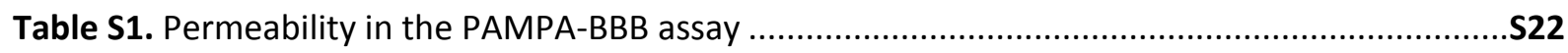


Figure S1. MS and NMR spectra of 11-O-propionylambelline (2)

388.17

20171116 LC-86 INJ1 1155 (1.982) Cm (1149:1166-(991:1082+1229:1290))

388.1757

1: TOF MS ES+

100

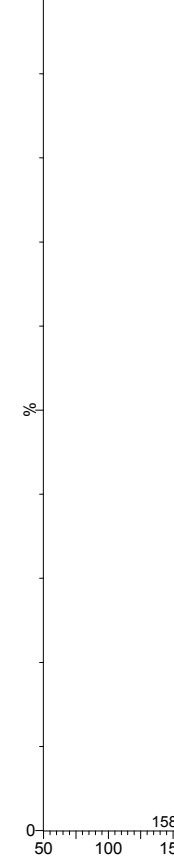

2

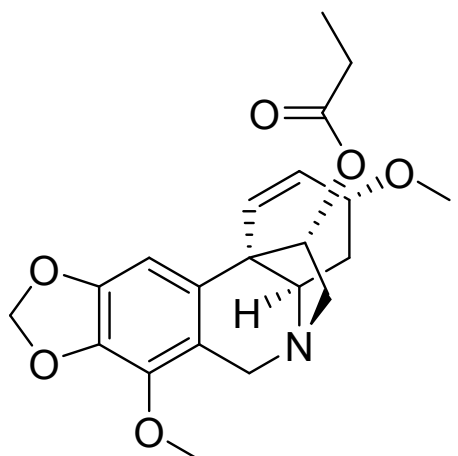

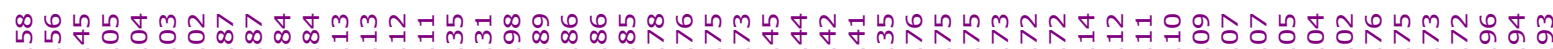

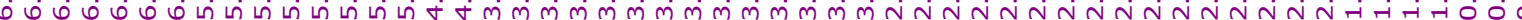

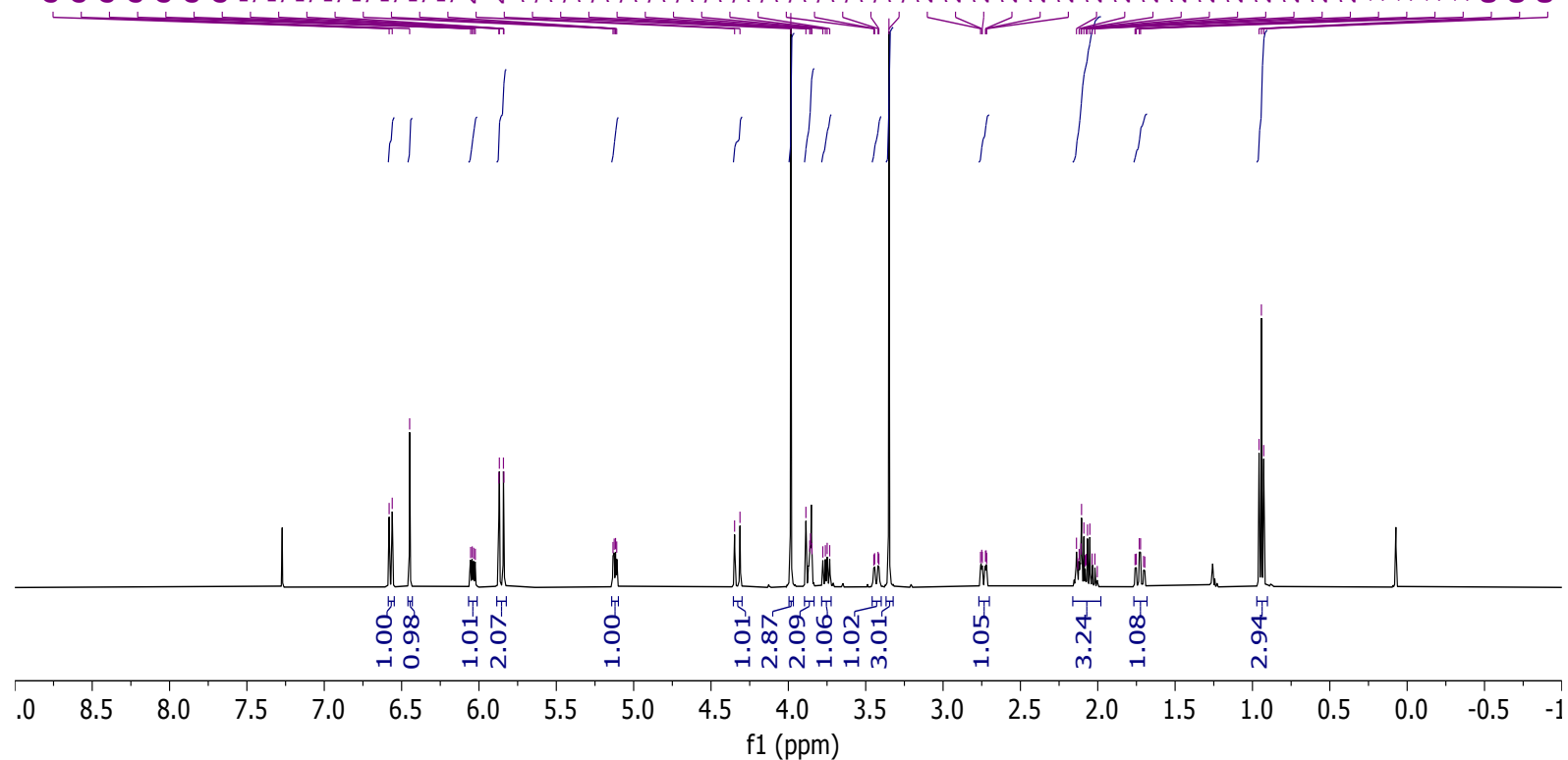

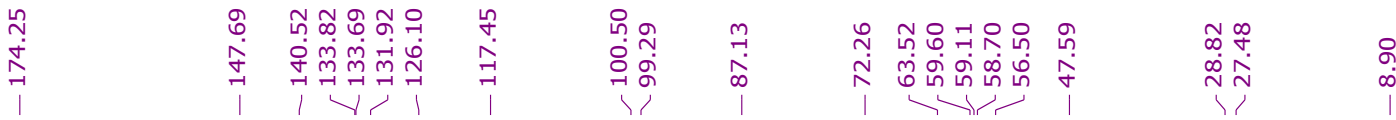

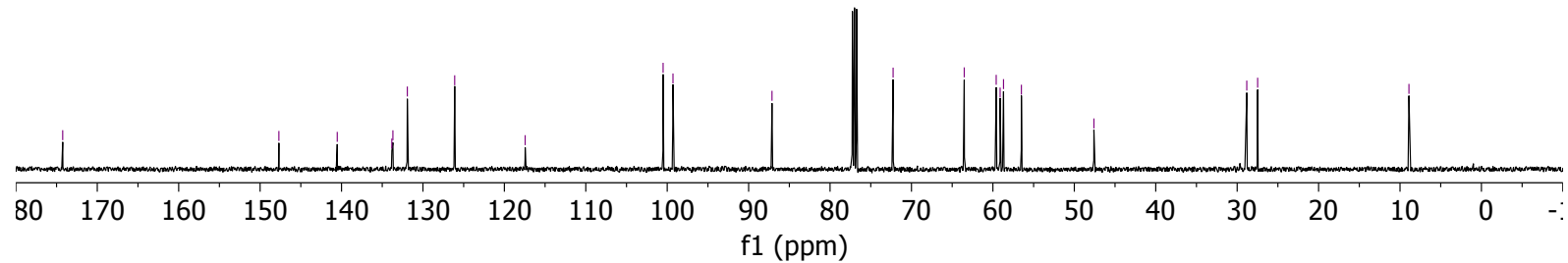


Figure S2. MS and NMR spectra of 11-O-pentanoylambelline (3)

416.20

20171116 LC-87 INJ1 1405 (2.410) Cm (1398:1415-(1220:1346+1503:1669))

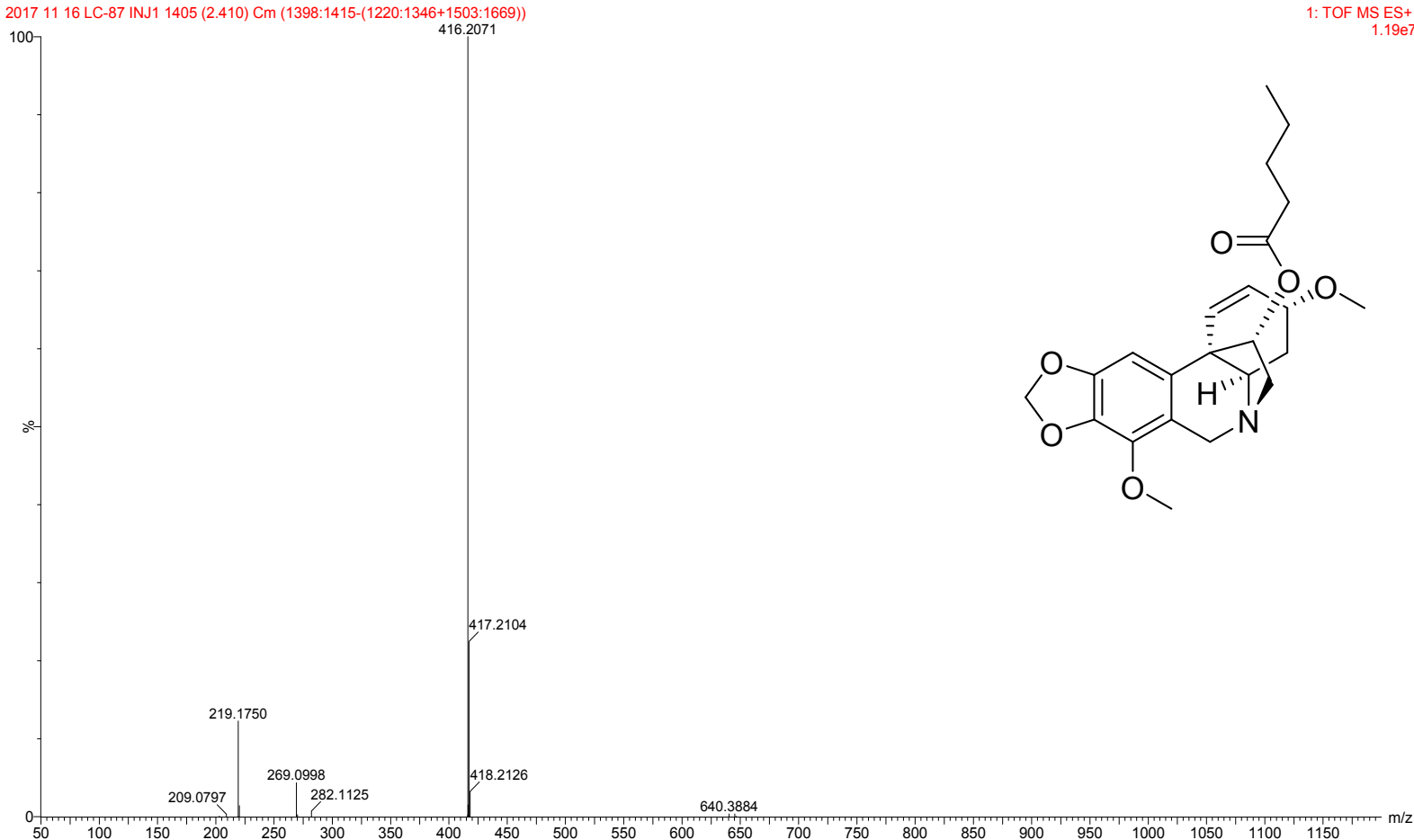

3

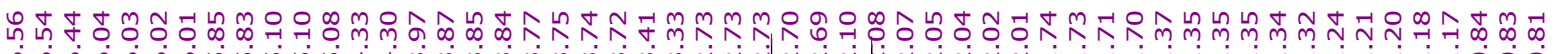

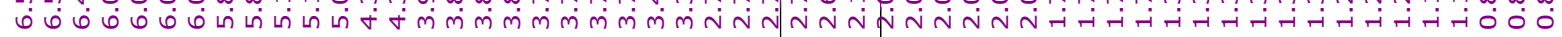

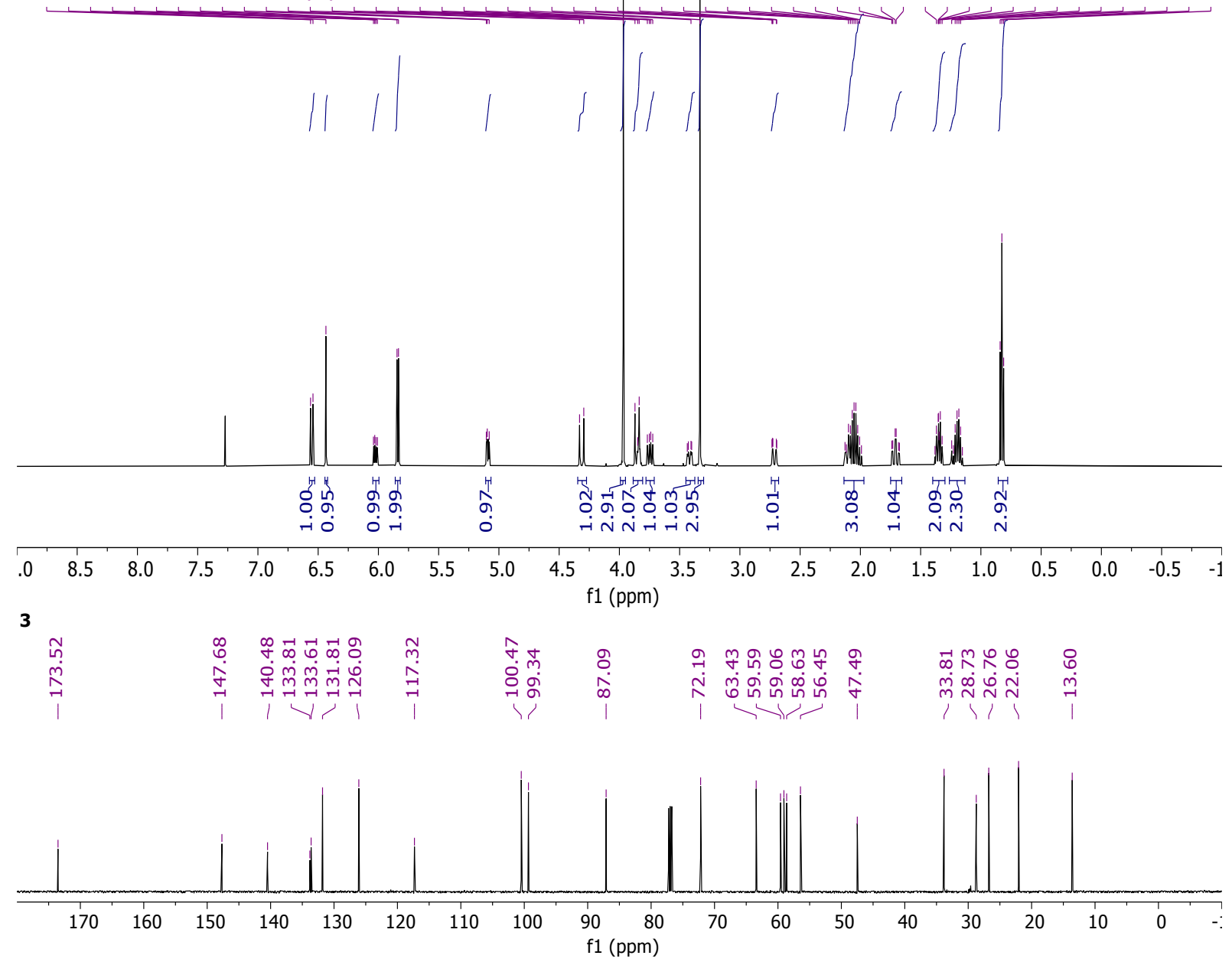


Figure S3. MS and NMR spectra of 11-O-isobutanoylambelline (4)

402.18

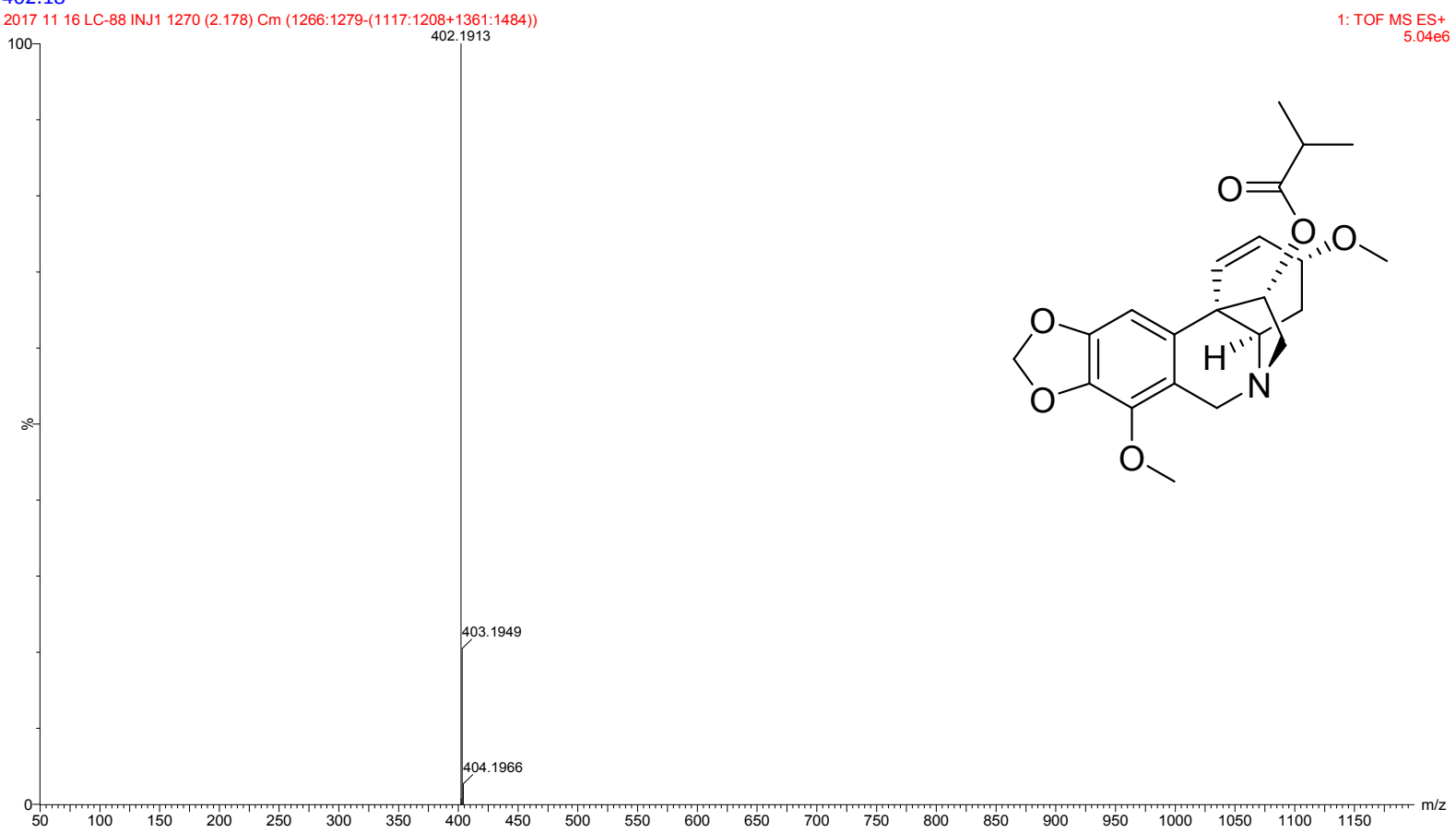

4

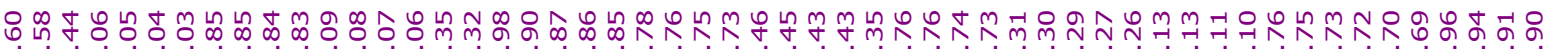

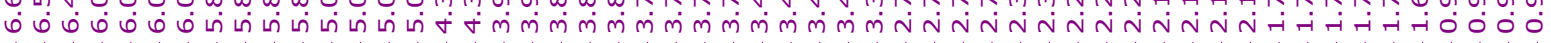

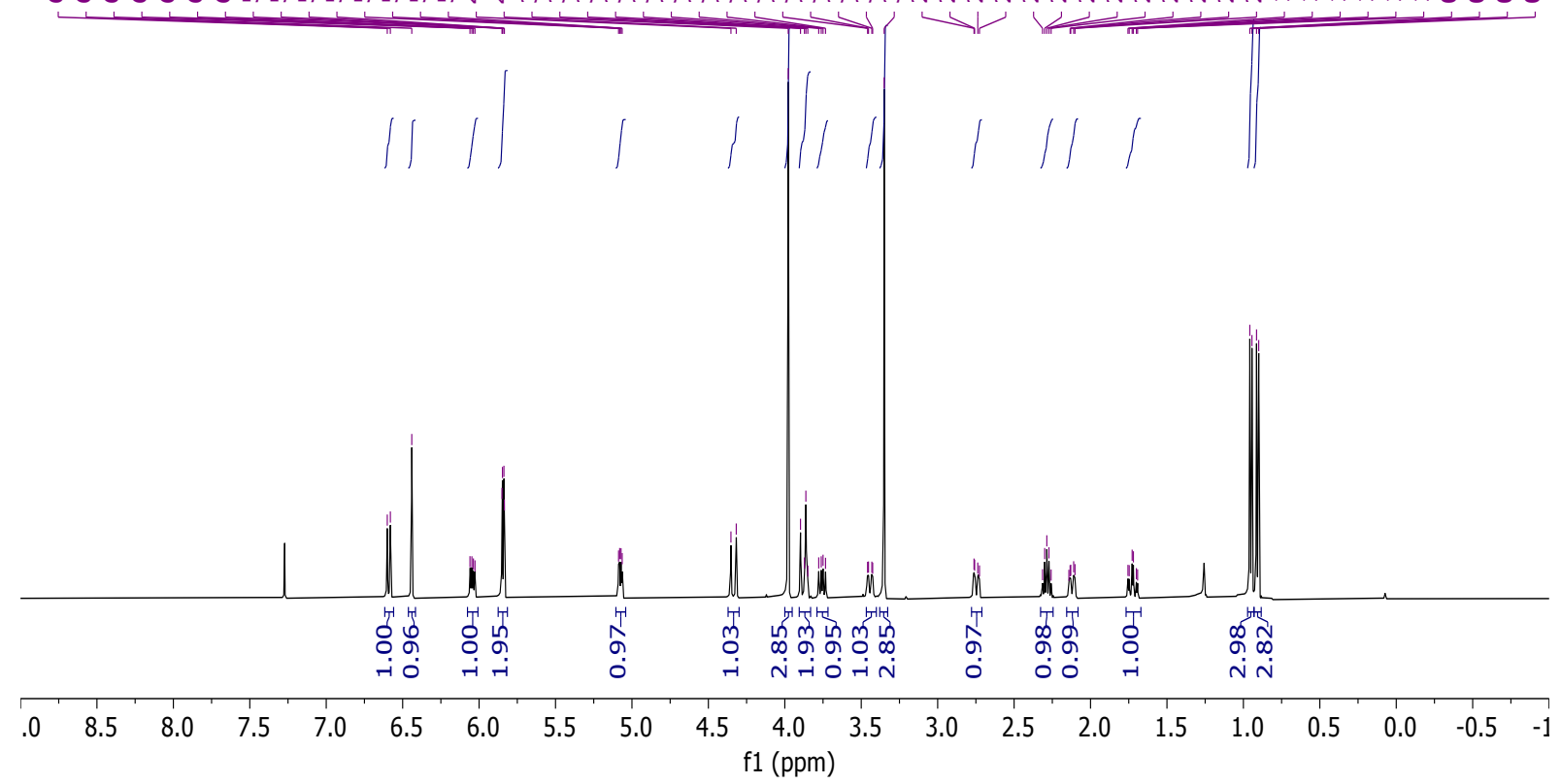

4

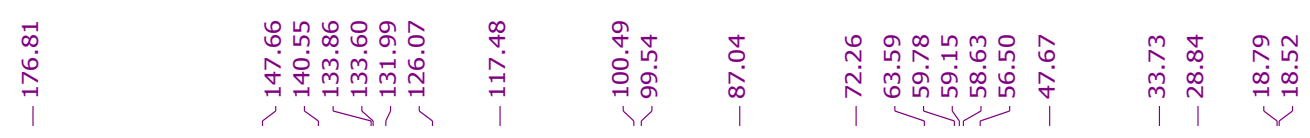

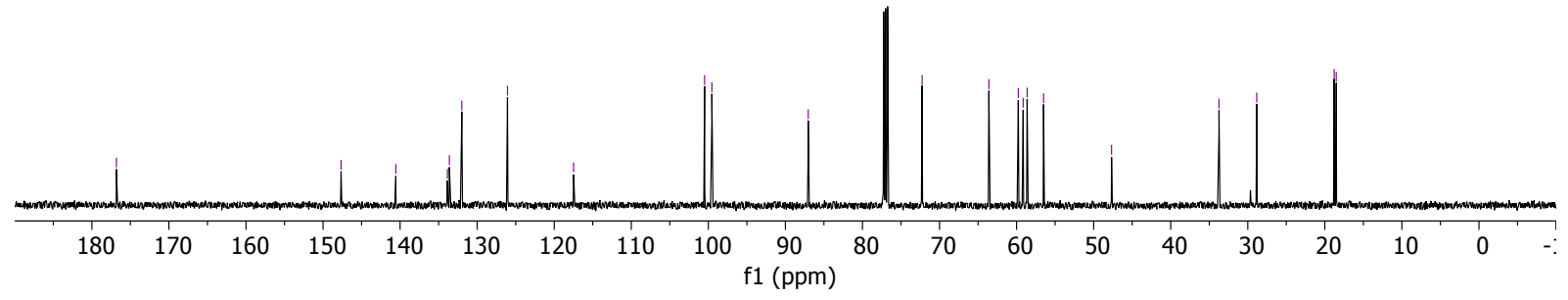


Figure S4. MS and NMR spectra of 11-O-benzoylambelline (5)

436.17

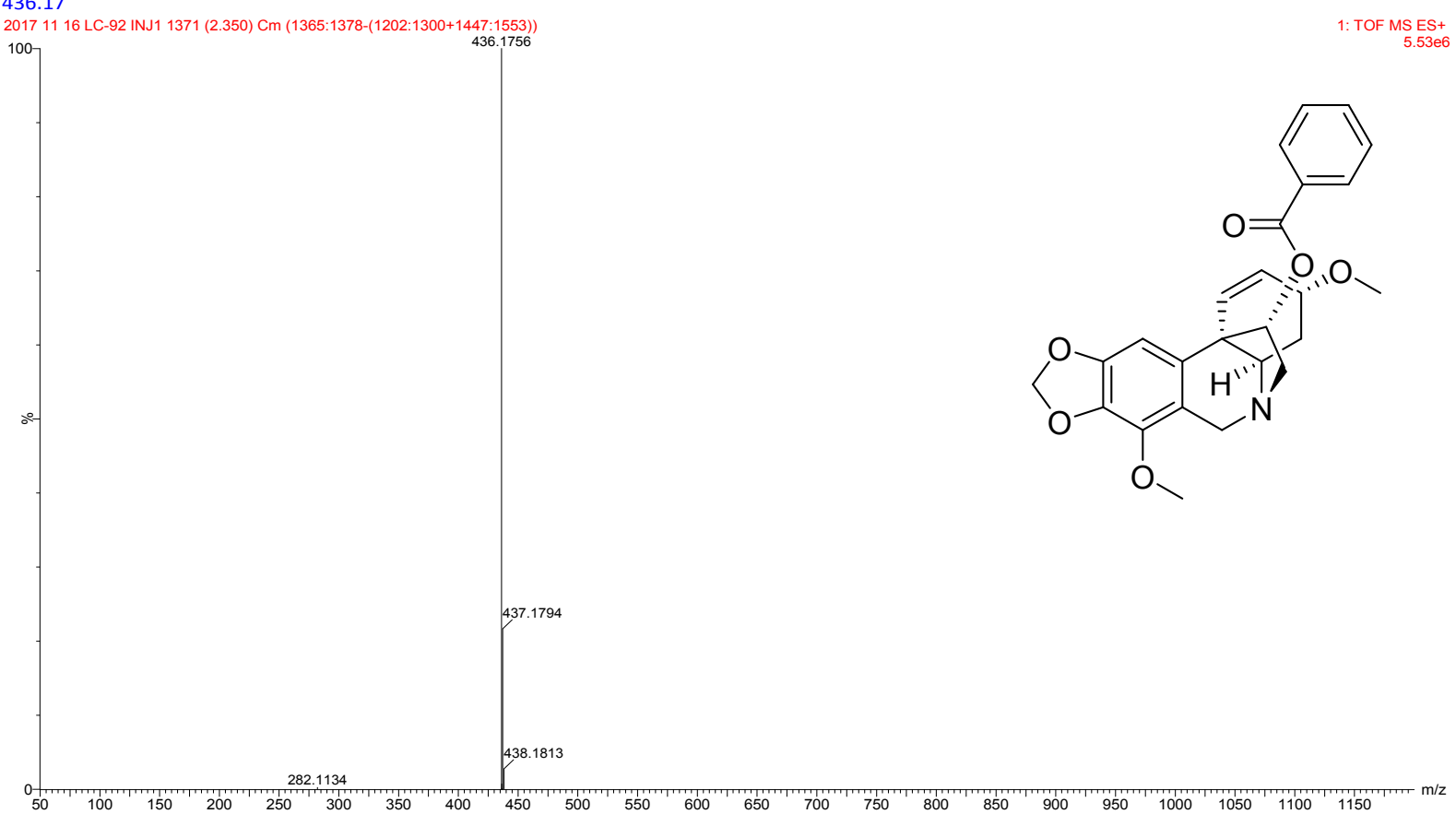

5

员

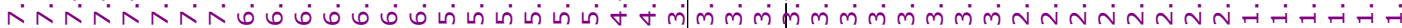

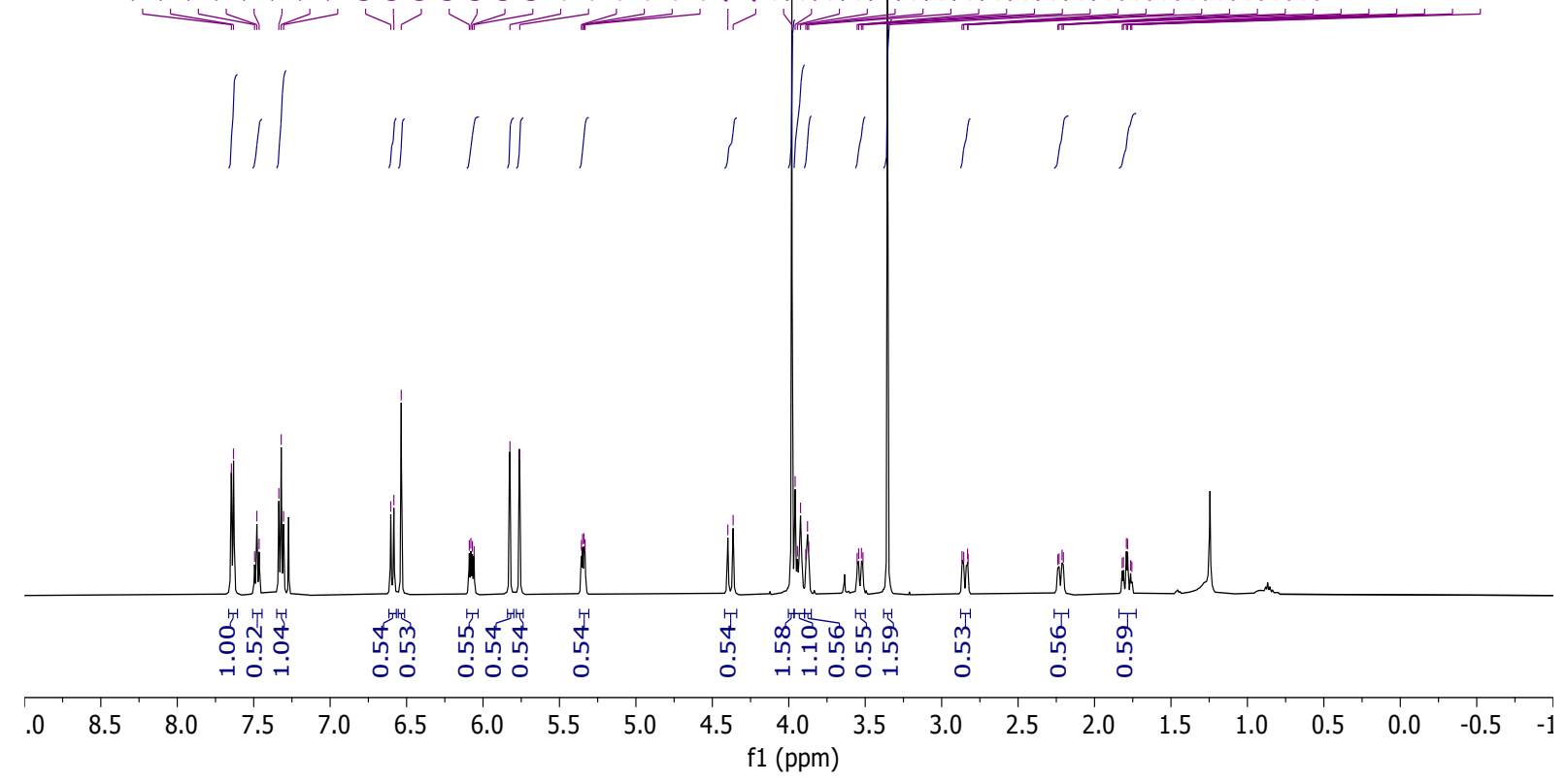

5

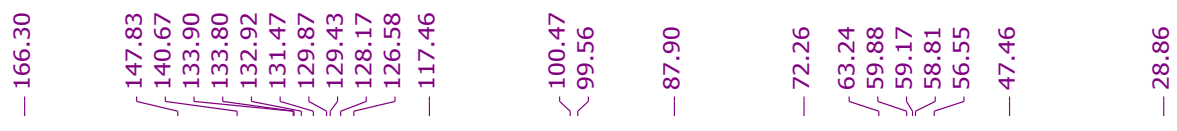

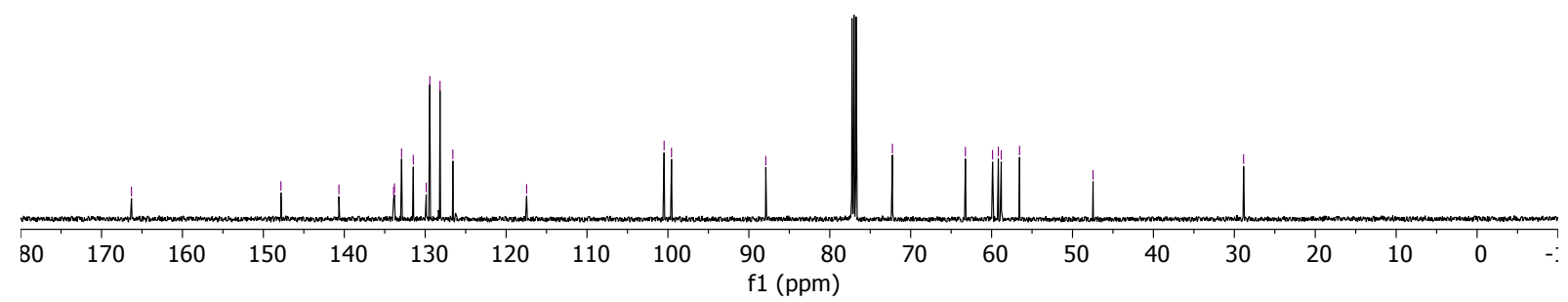


Figure S5. MS and NMR spectra of 11-O-(2-methylbenzoyl)ambelline (6)

450.1917

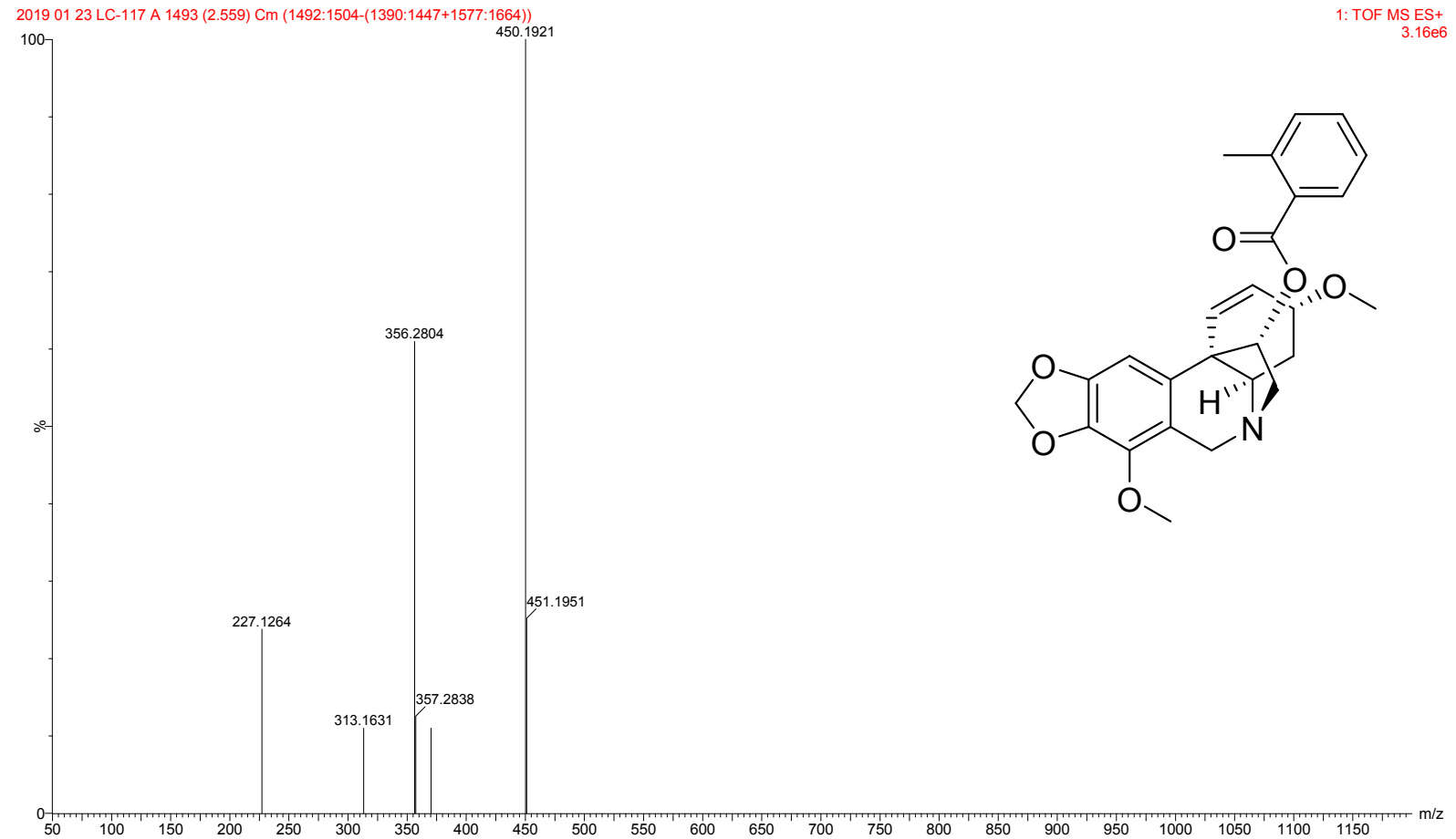

6

m户

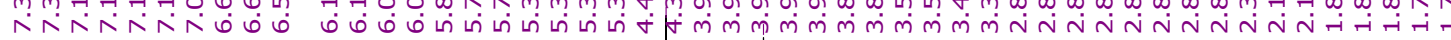

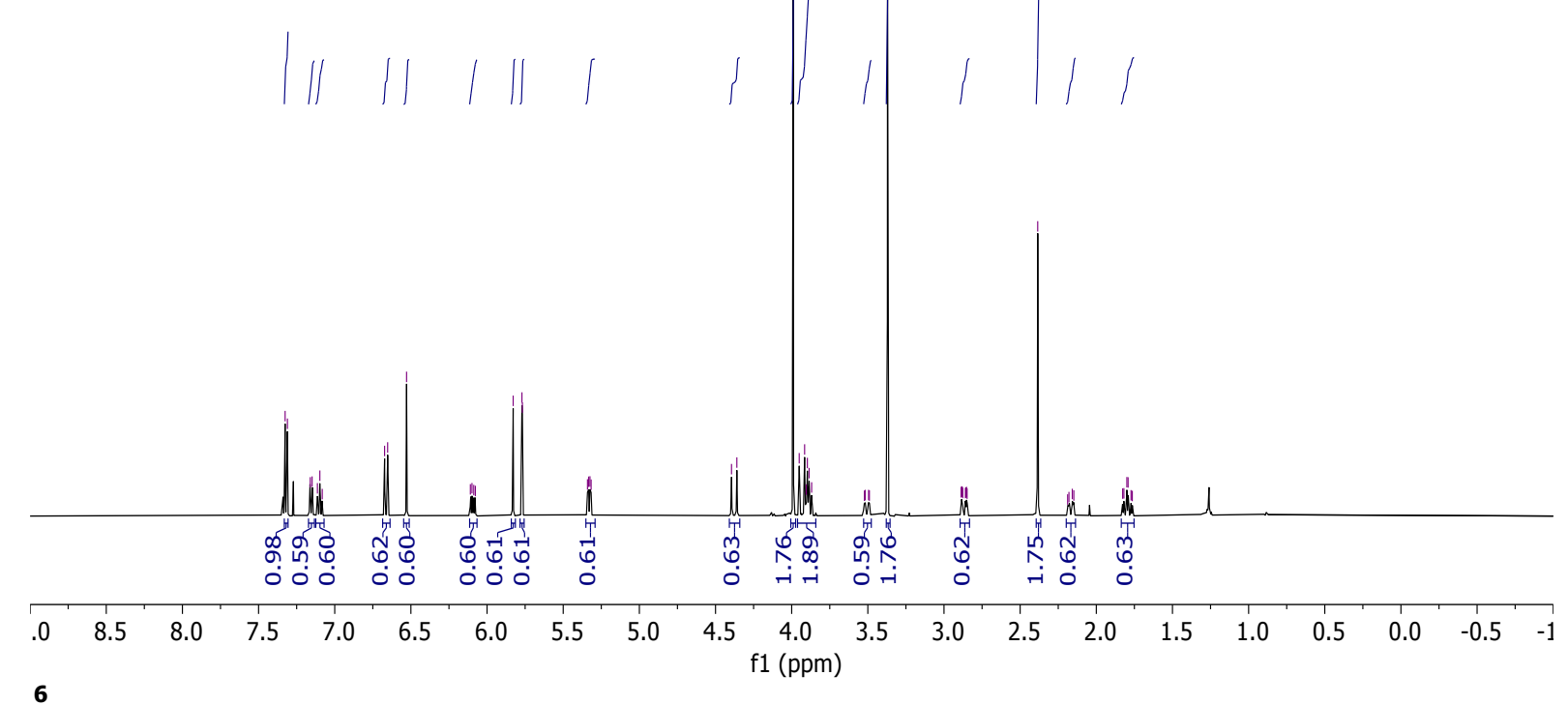

6

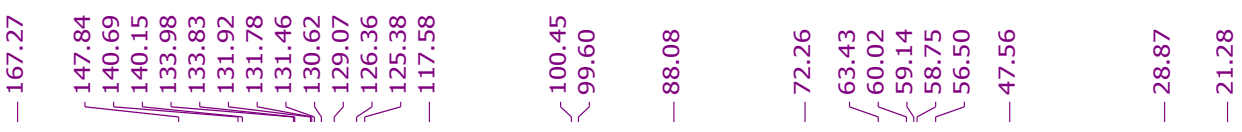

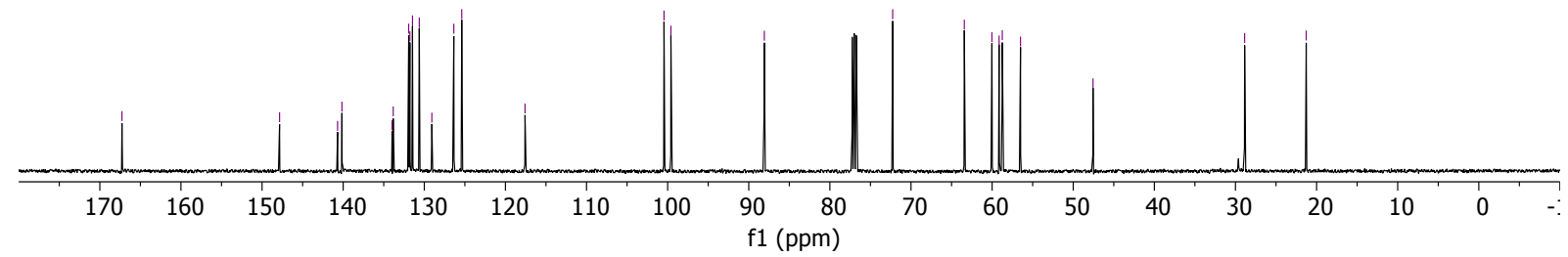


Figure S6. MS and NMR spectra of 11-O-(3-methylbenzoyl)ambelline (7)

450.1917

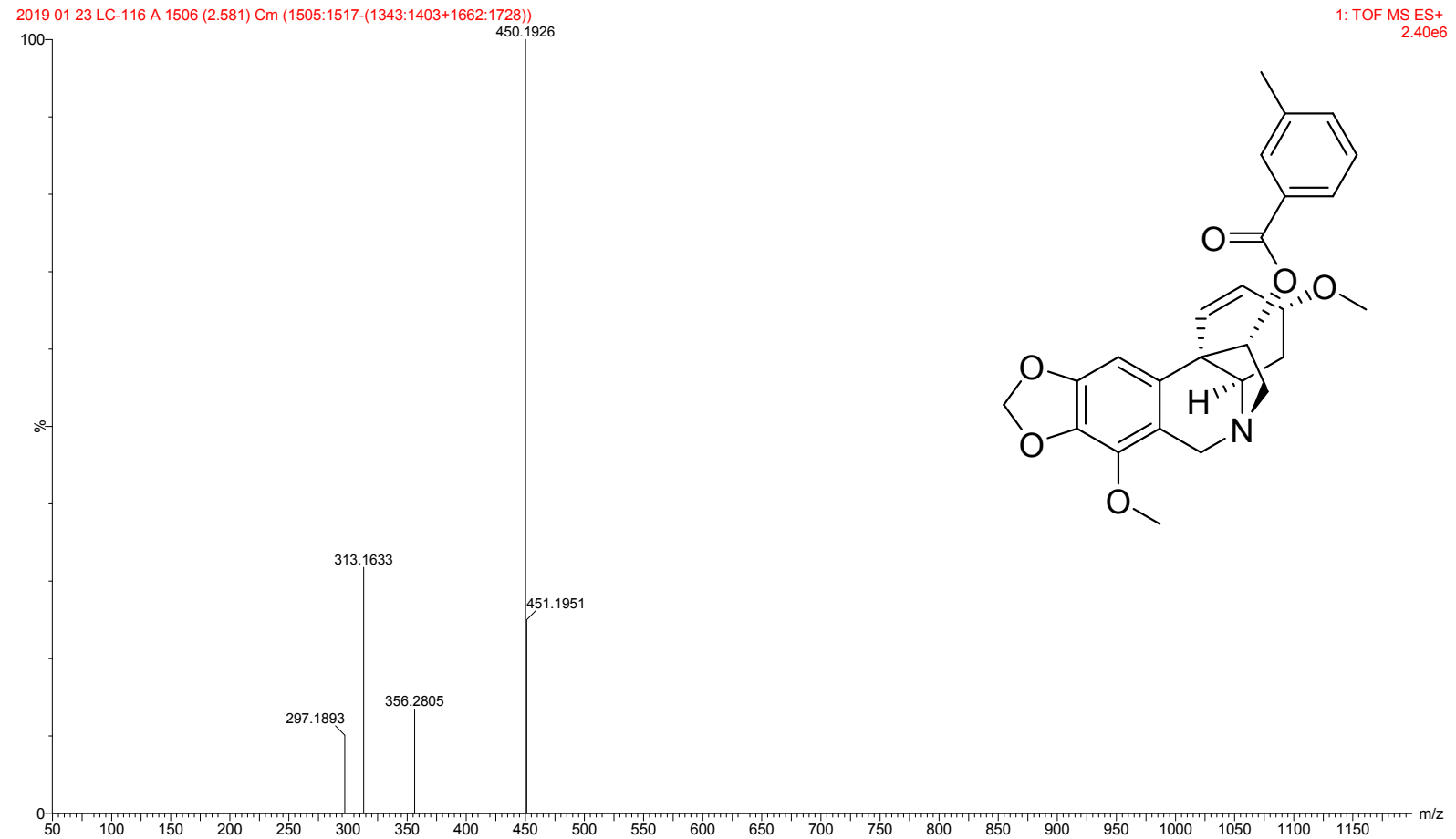

7

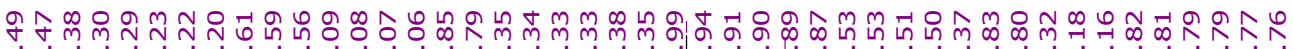

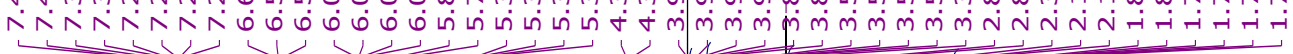

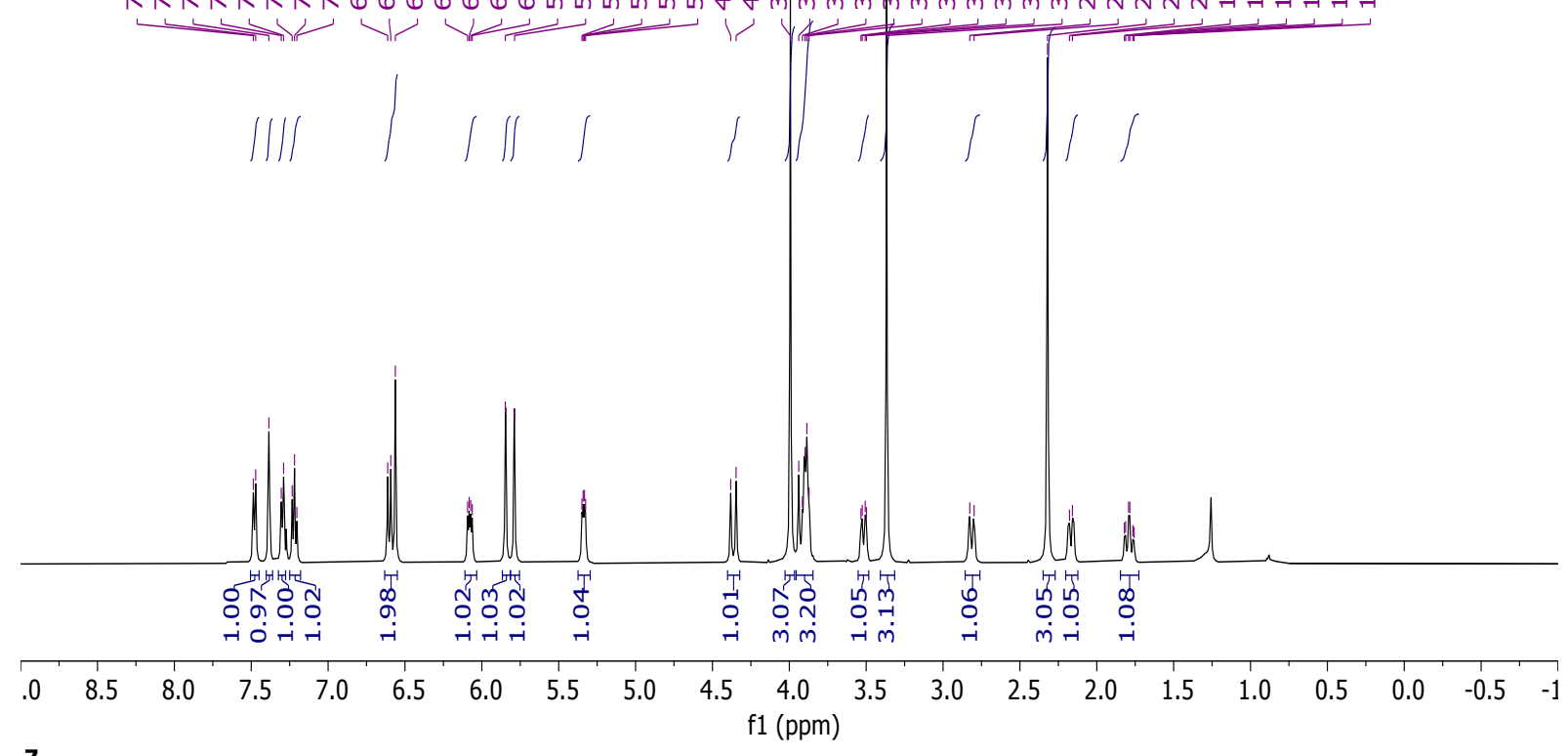

7

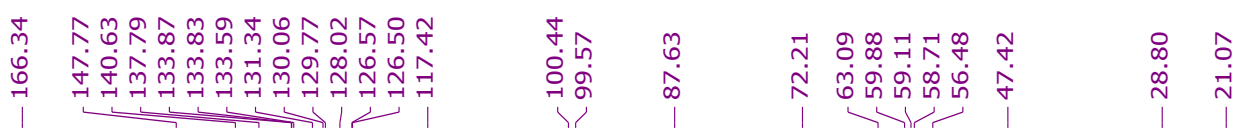

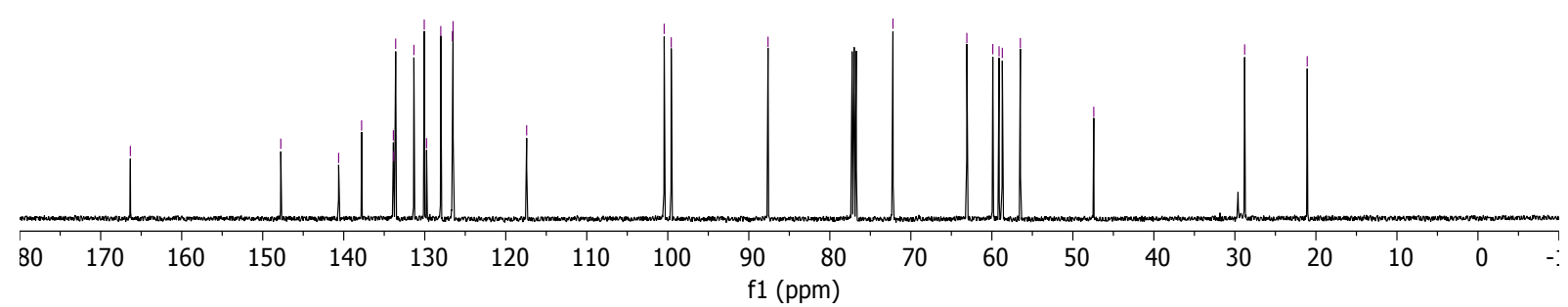


Figure S7. MS and NMR spectra of 11-O-(4-methylbenzoyl)ambelline (8)

450.1917

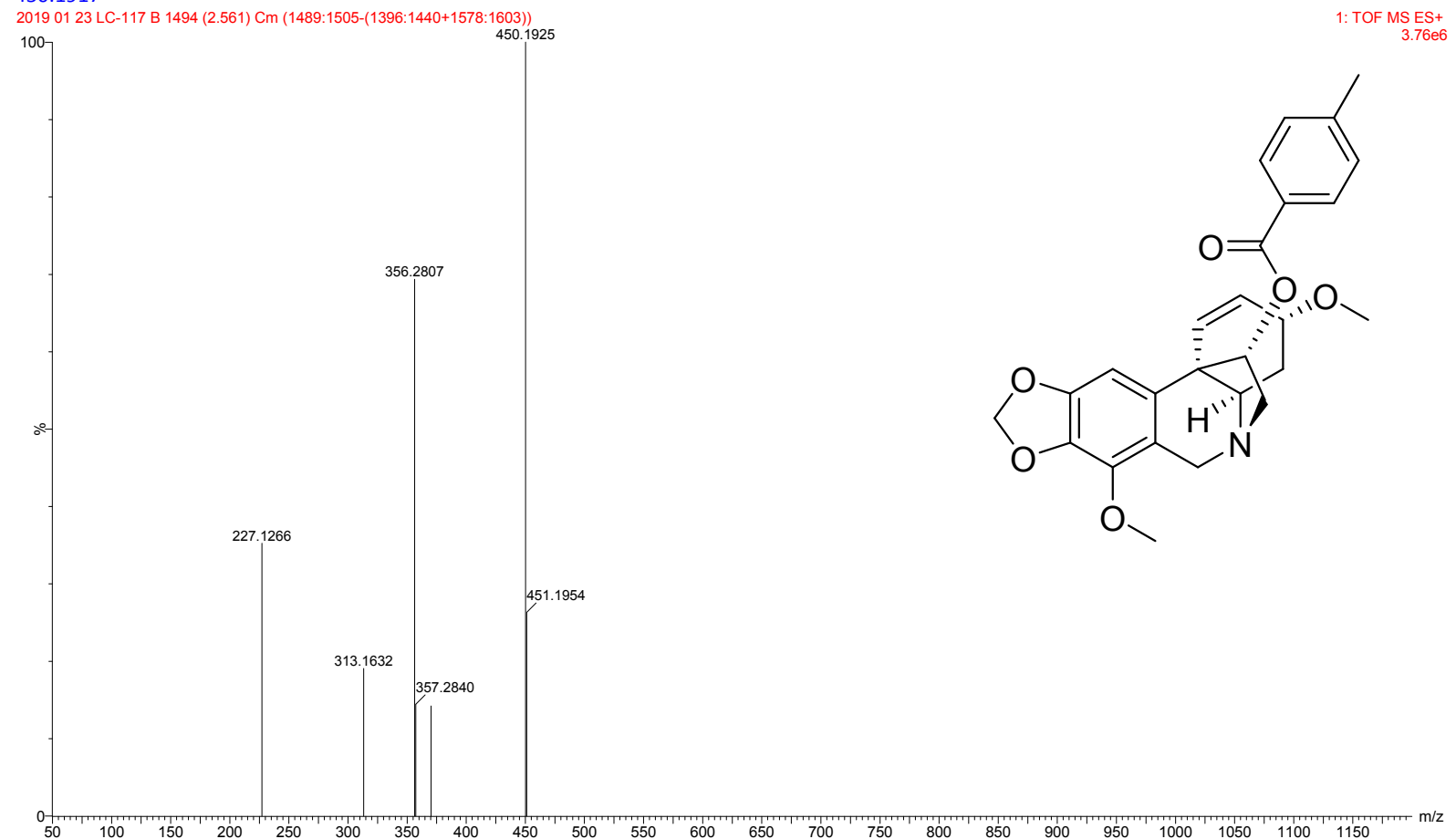

8

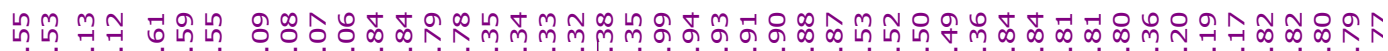

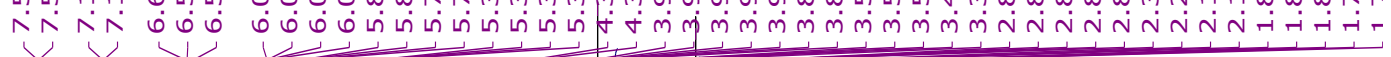

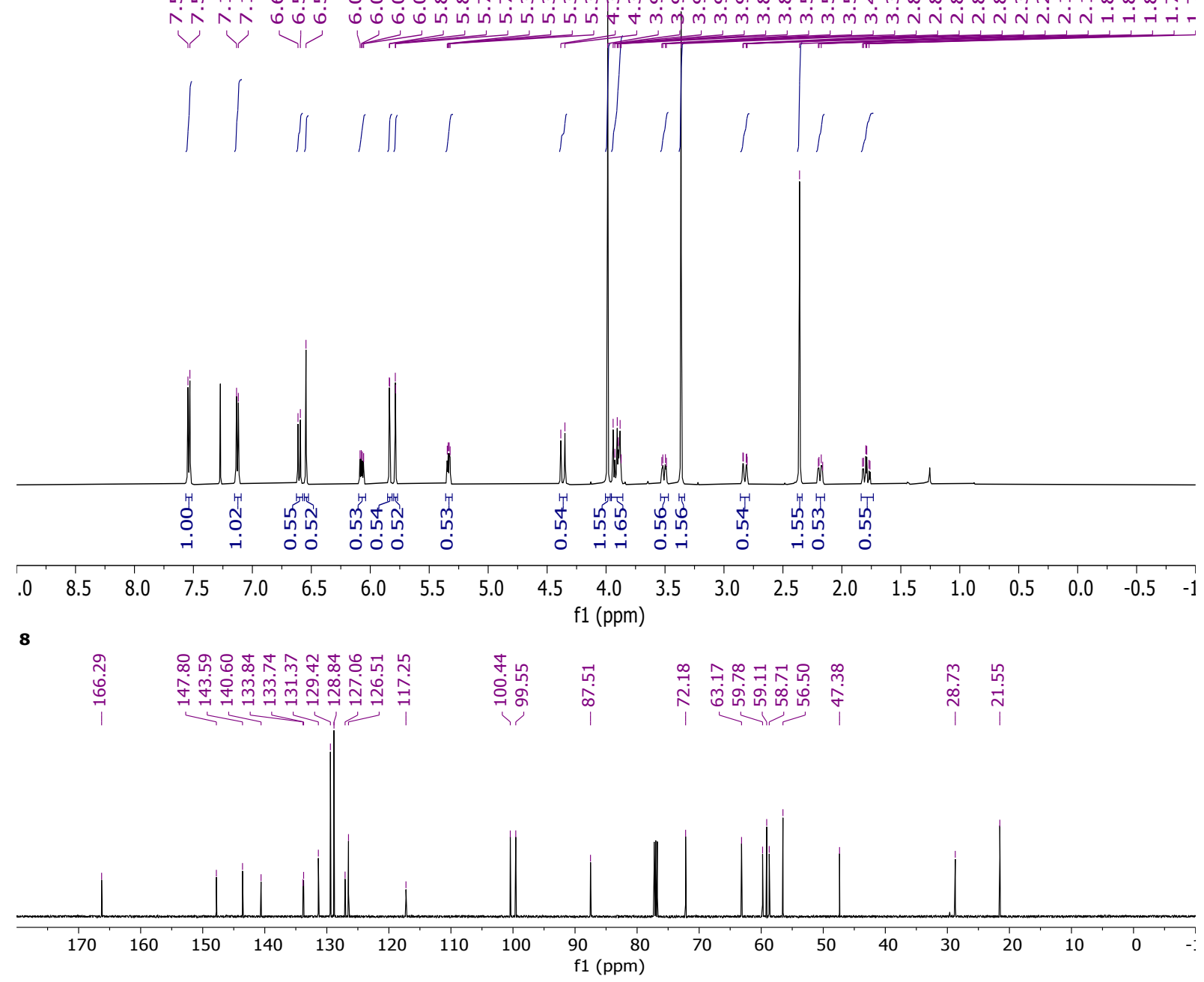


Figure S8. MS and NMR spectra of 11-O-(2-methoxybenzoyl)ambelline (9)

466.1788, 10-7

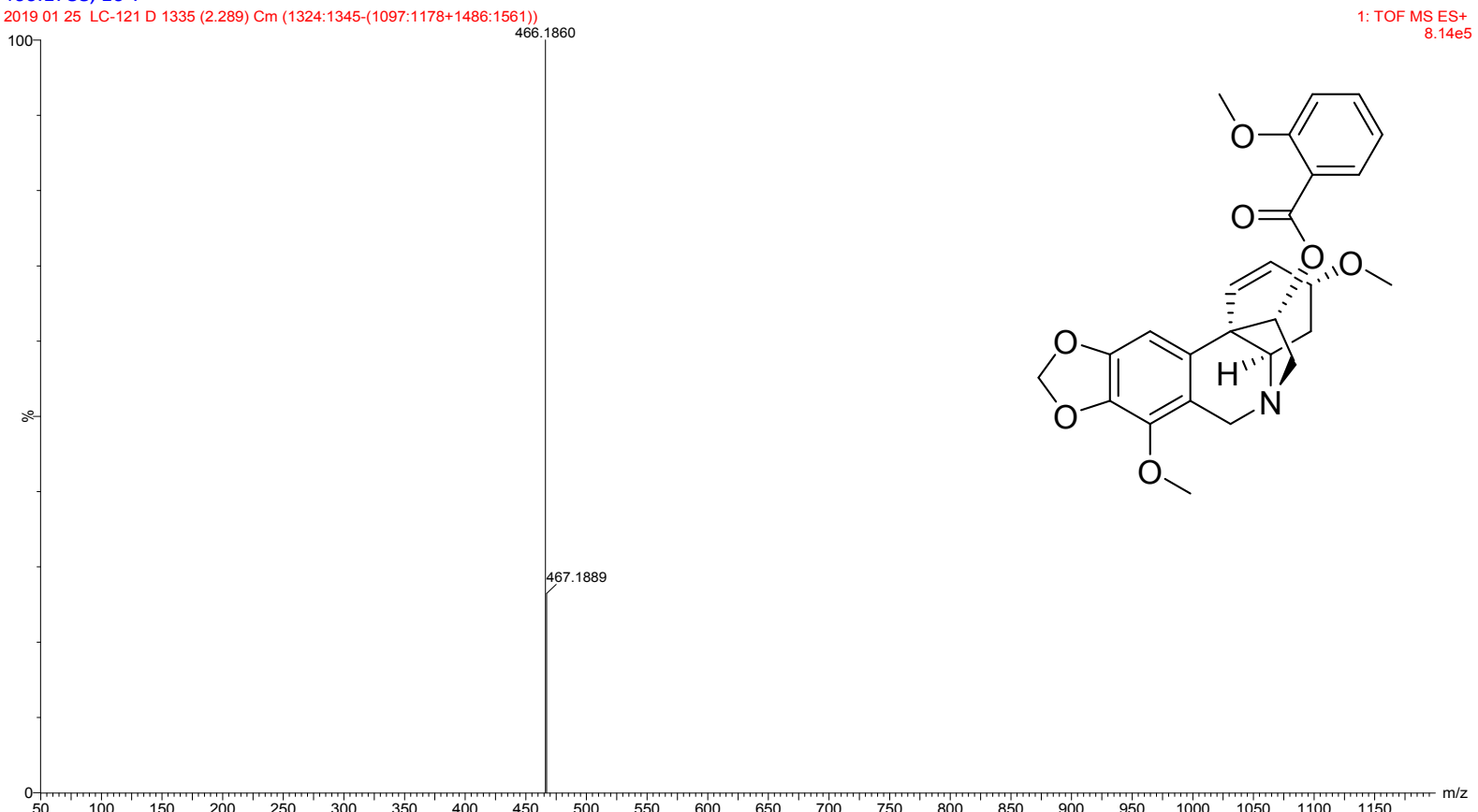

9

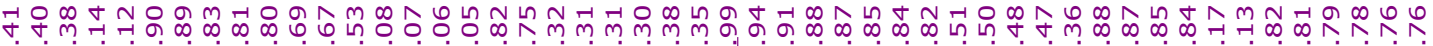

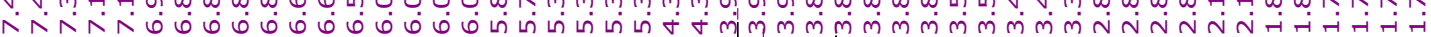

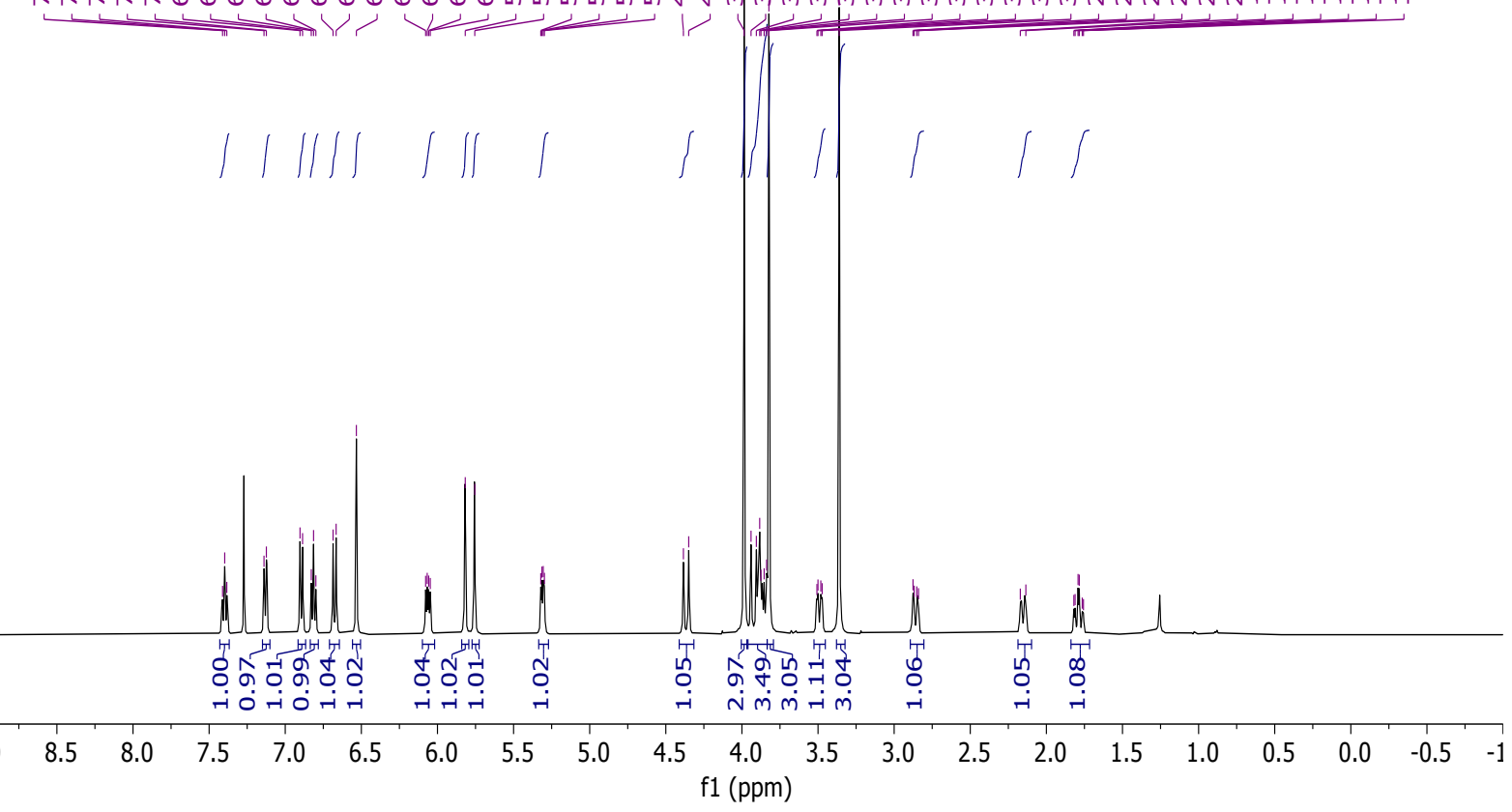

9

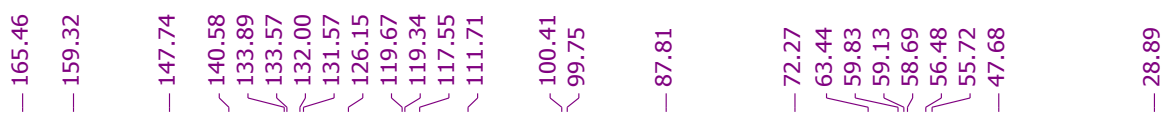

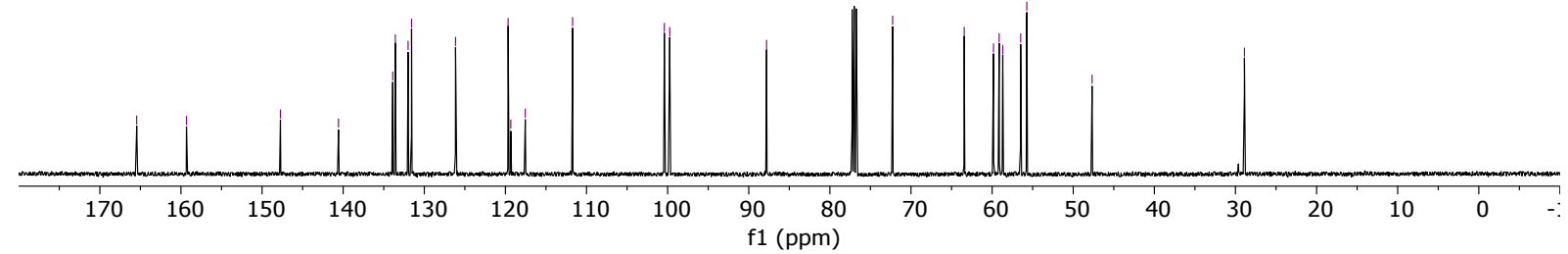


Figure S9. MS and NMR spectra of 11-O-(3-methoxybenzoyl)ambelline (10)

\subsection{8, 10-7}

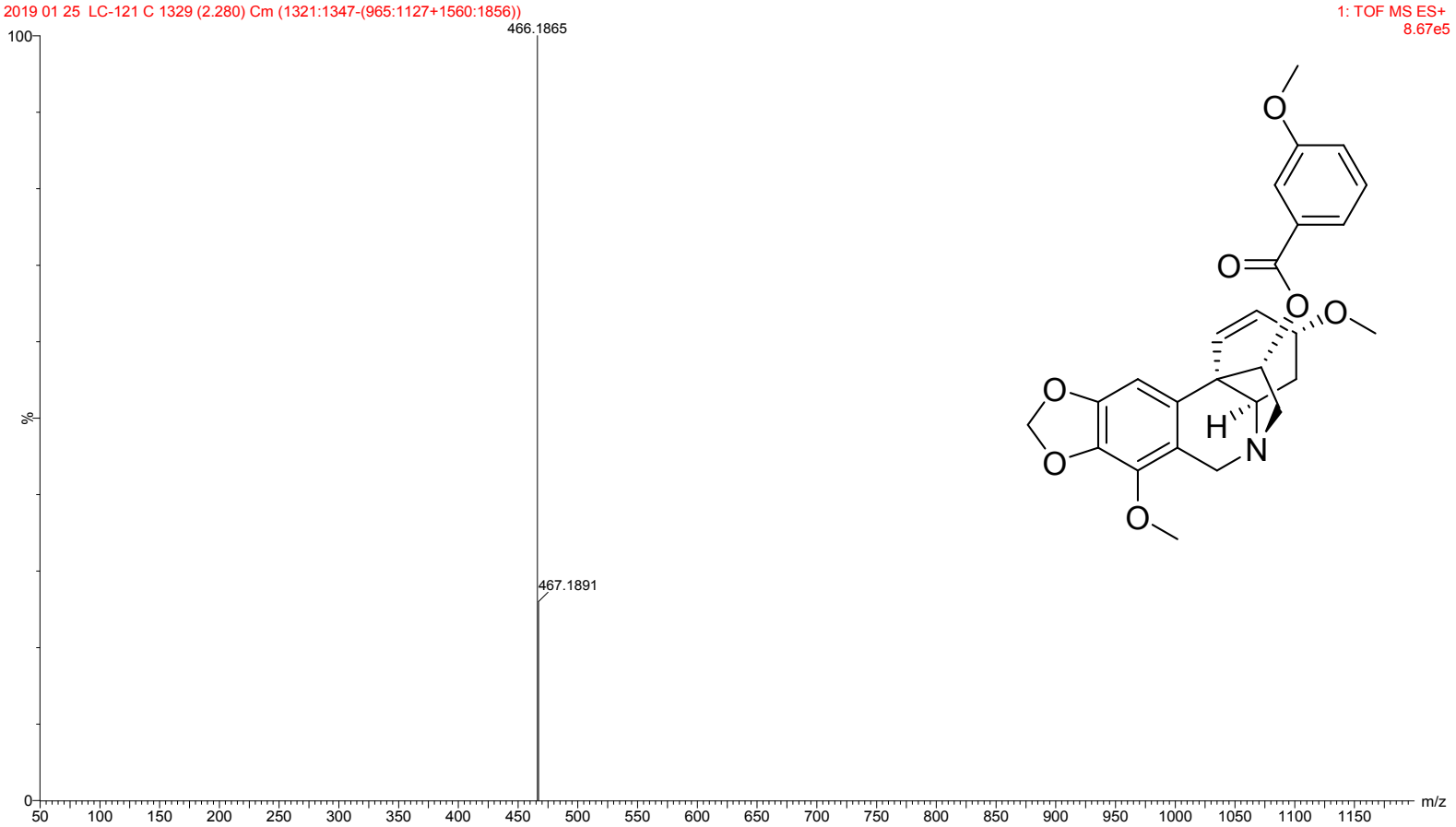

10

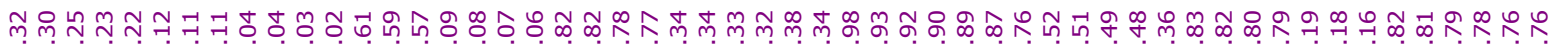

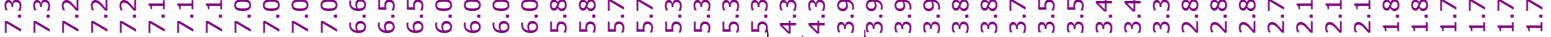

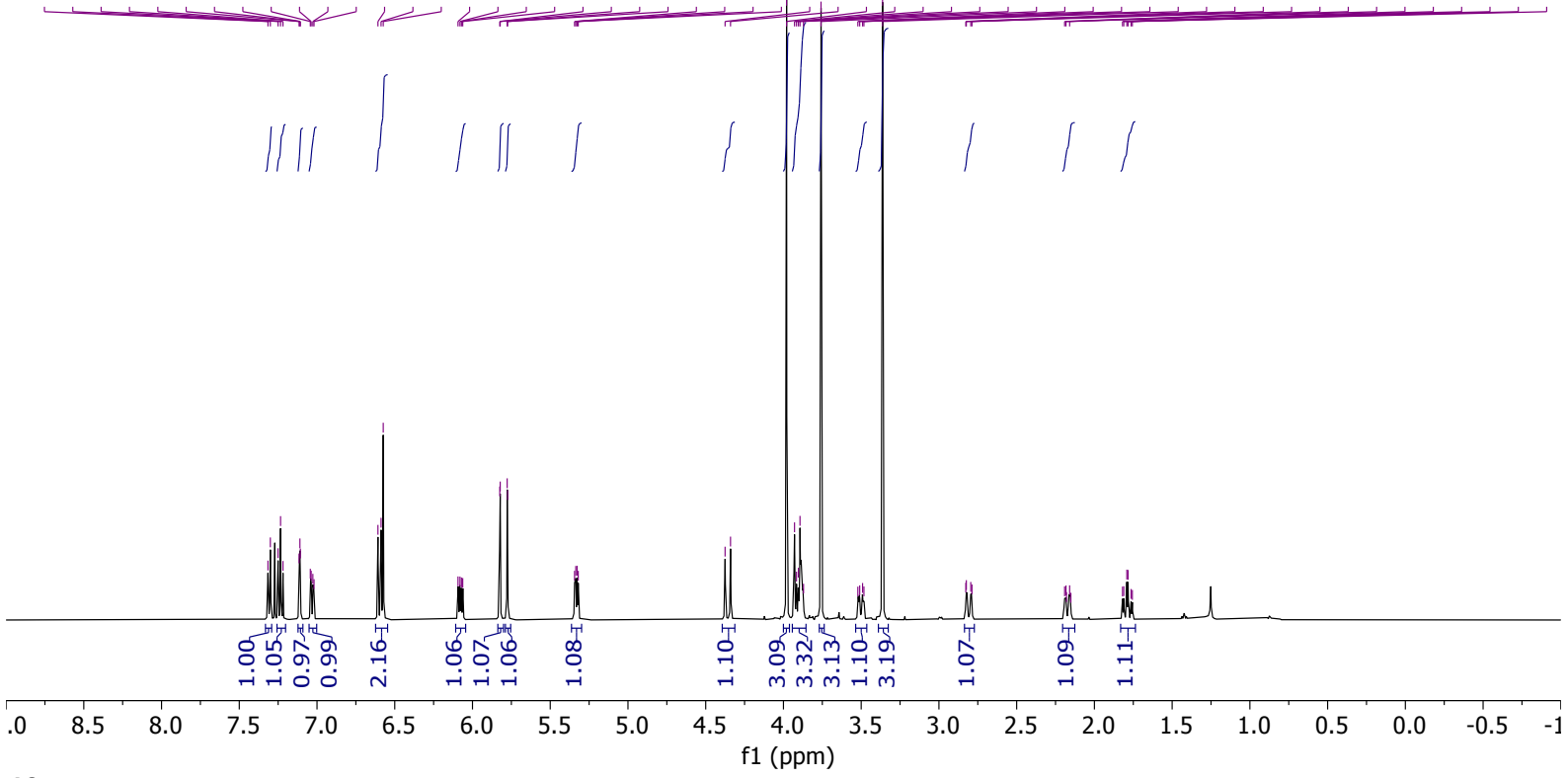

10

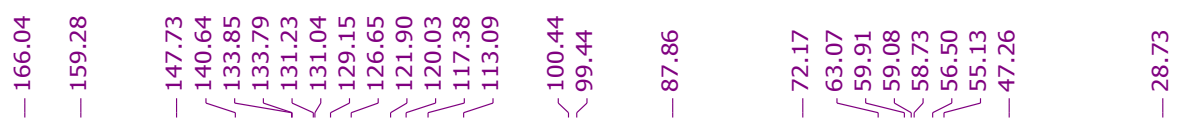

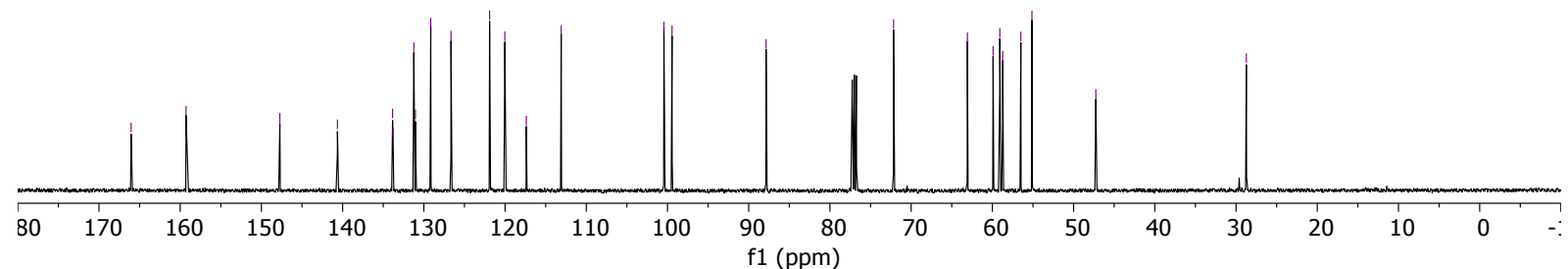


Figure S10. MS and NMR spectra of 11-O-(4-methoxybenzoyl)ambelline (11)

466.1788, 10-7

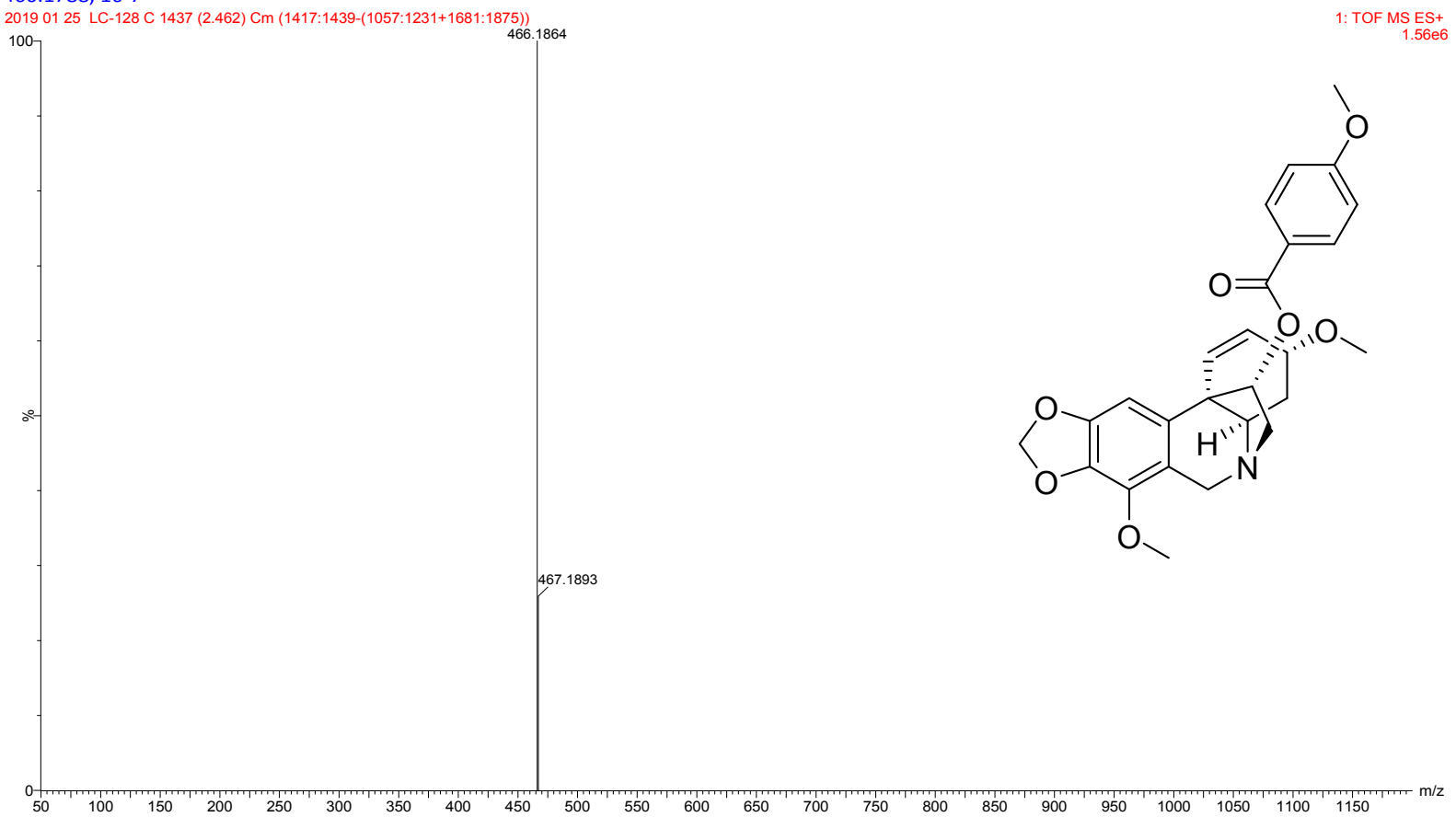

11

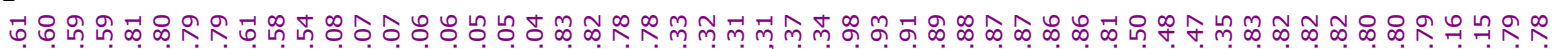

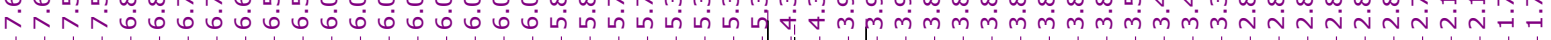

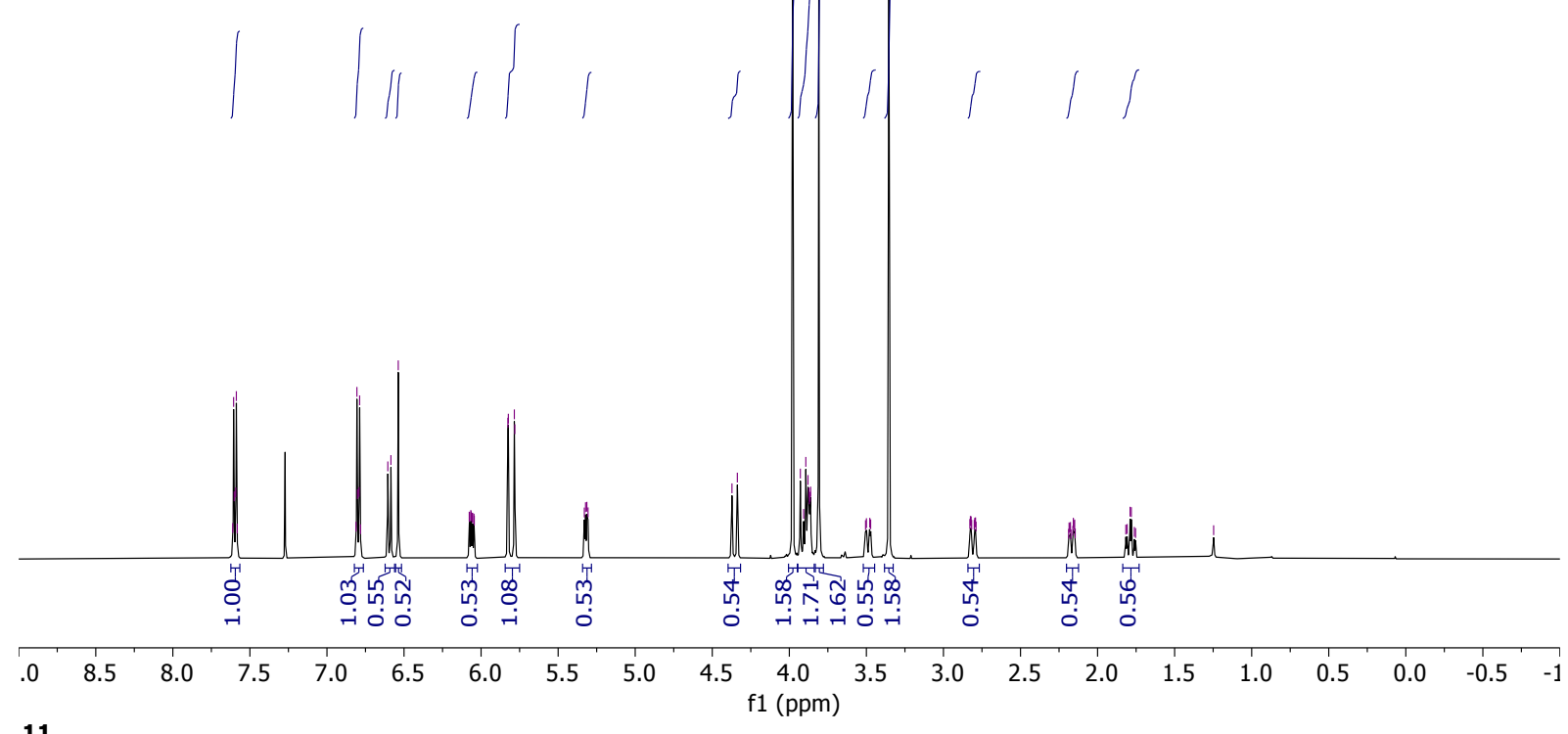

11

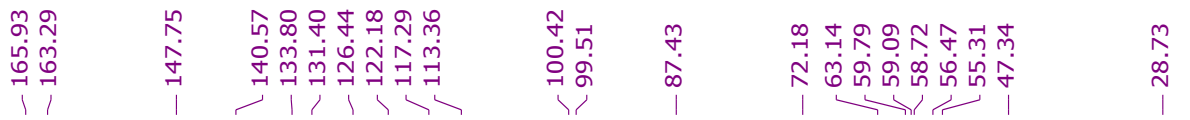

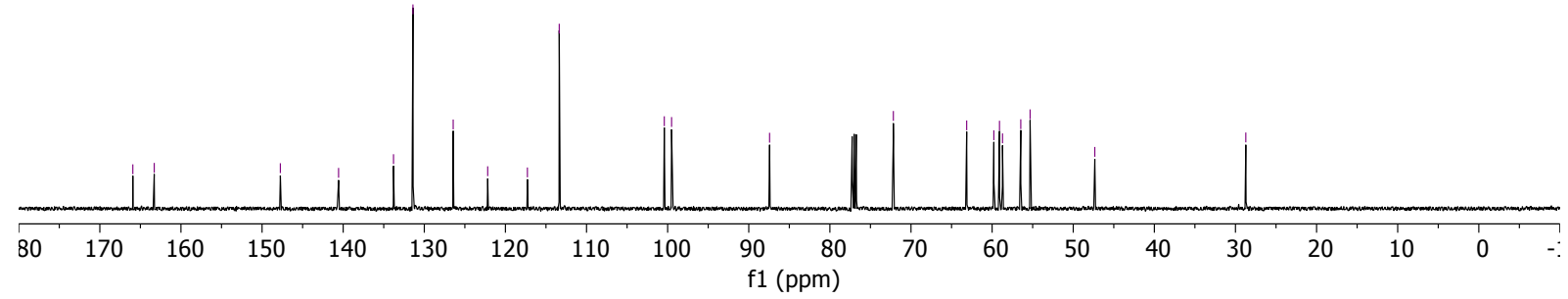


Figure S11. MS and NMR spectra of 11-O-(2-nitrobenzoyl)ambelline (12)

481.1611

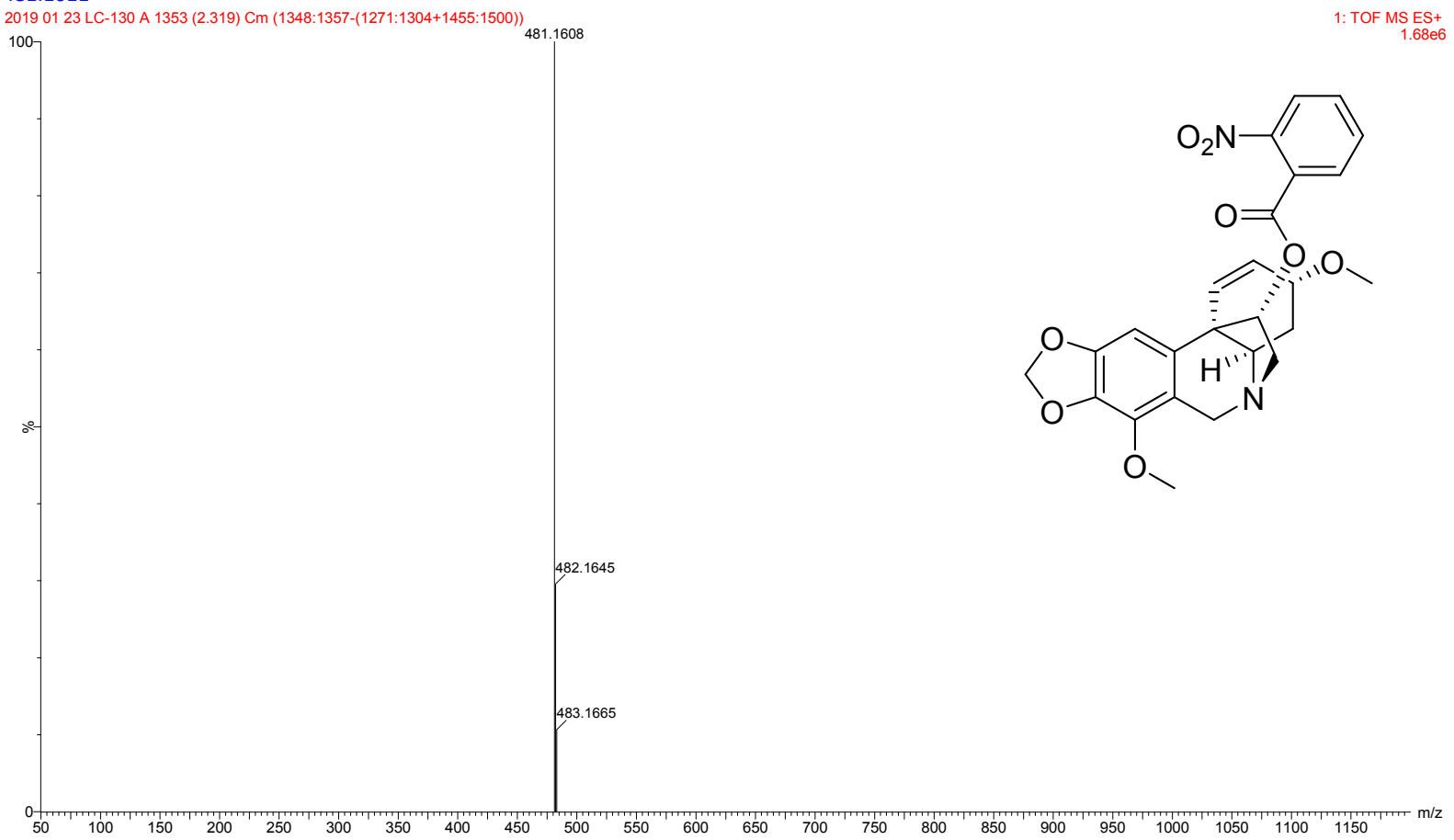

12

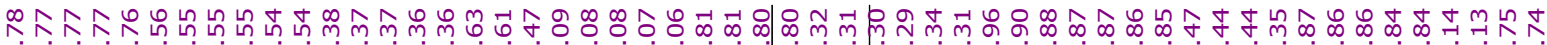

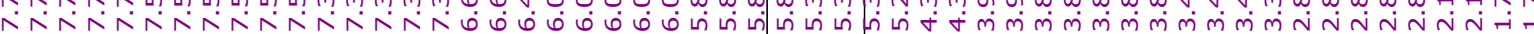

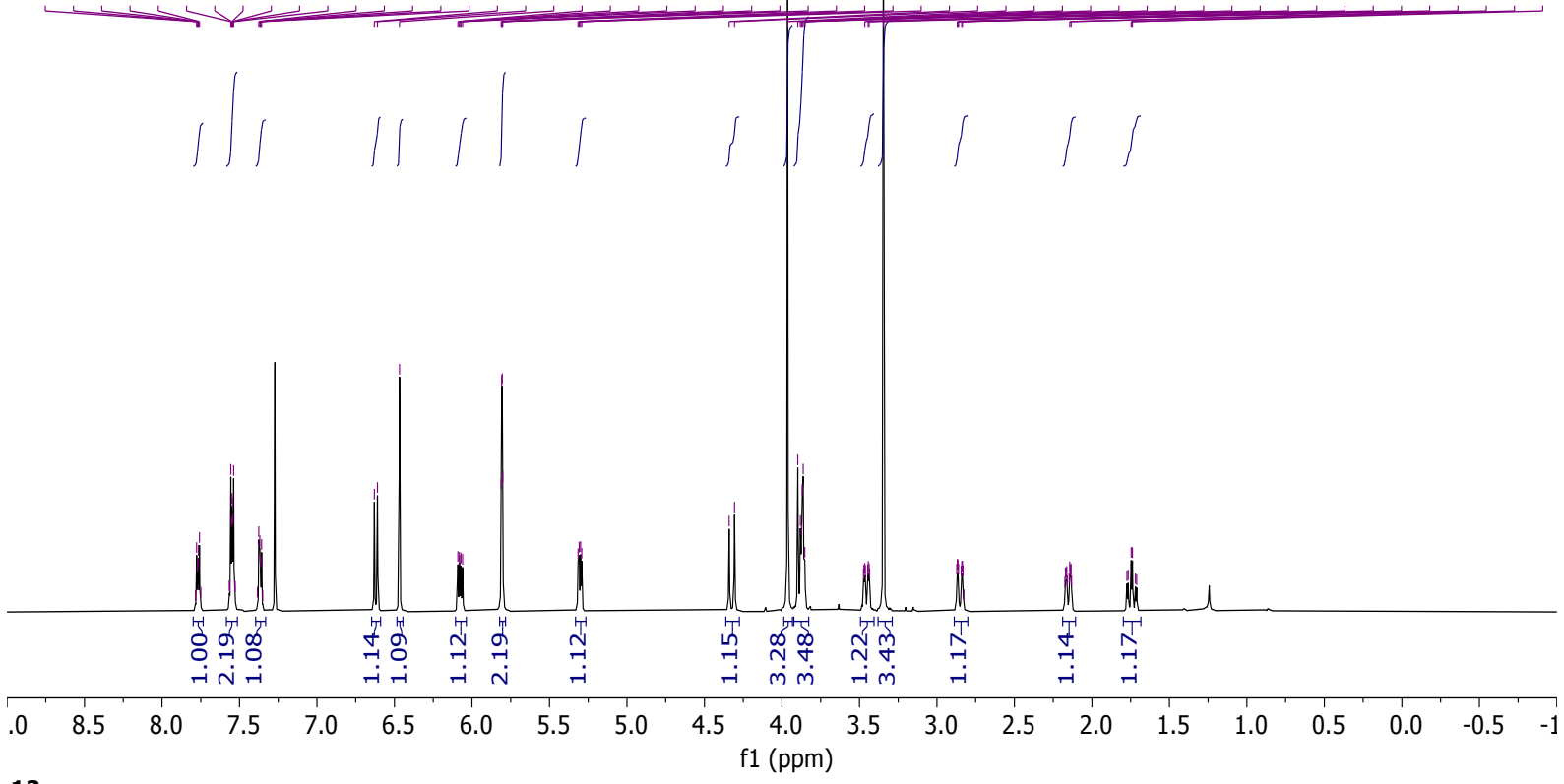

12

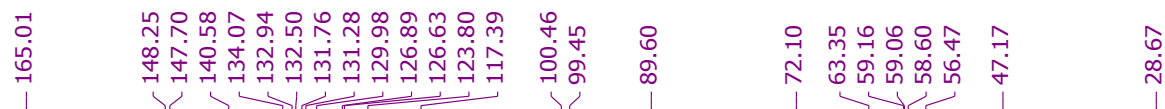

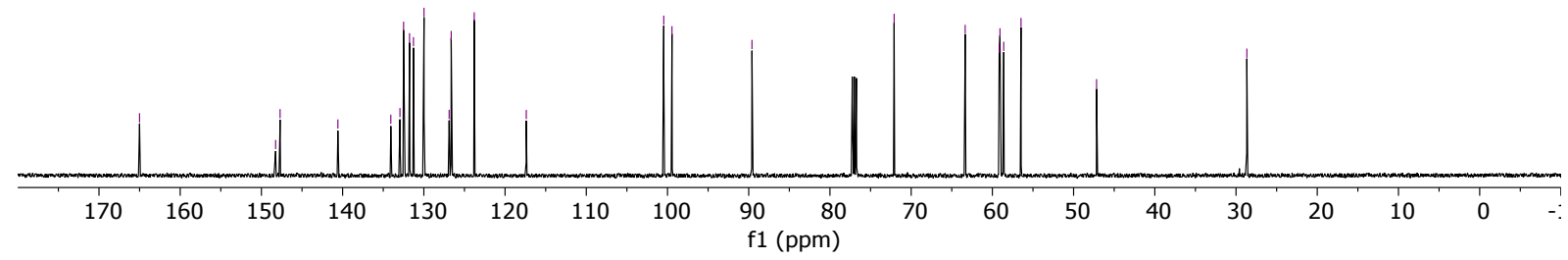


Figure S12. MS and NMR spectra of 11-O-(3-nitrobenzoyl)ambelline (13)

481.15

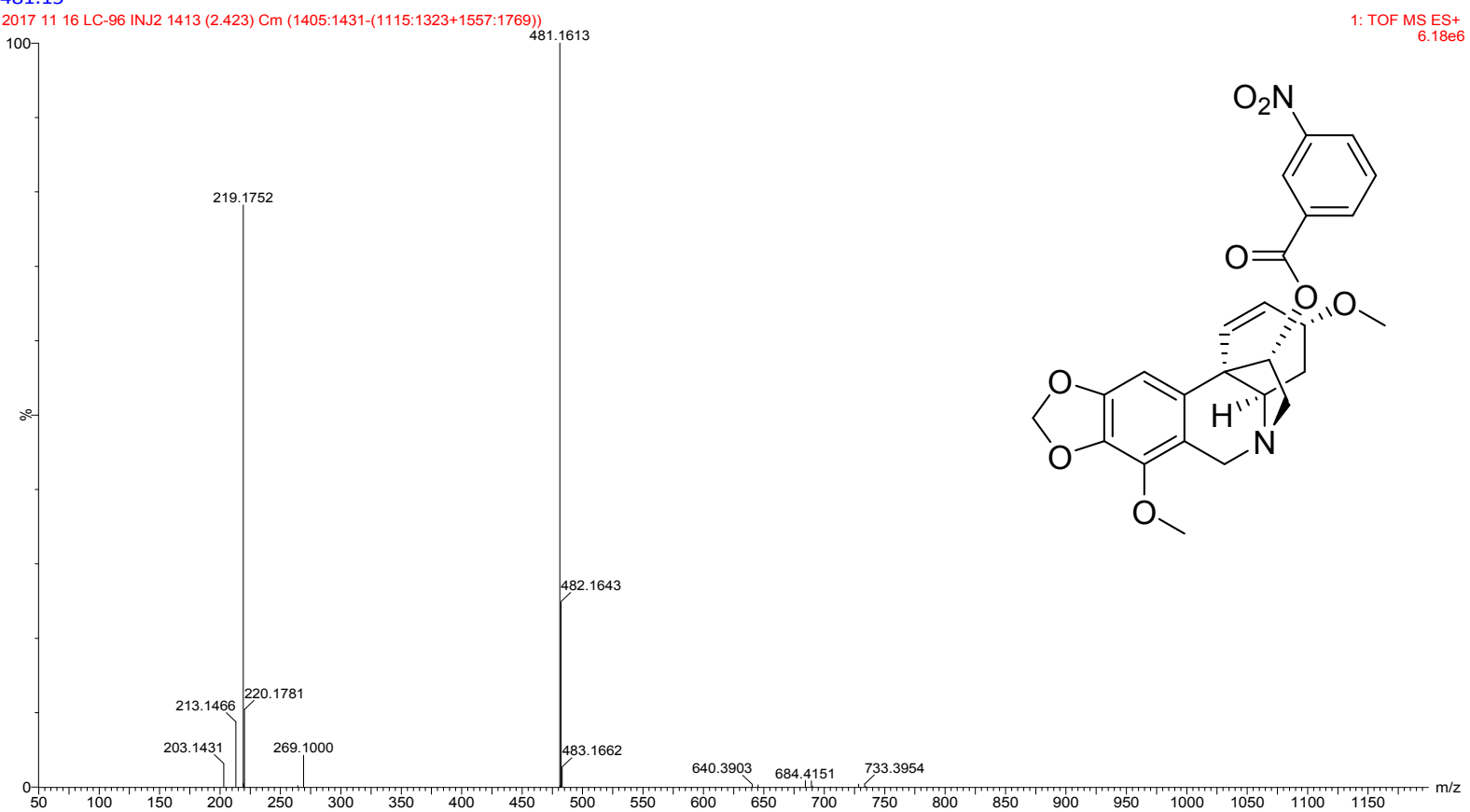

13

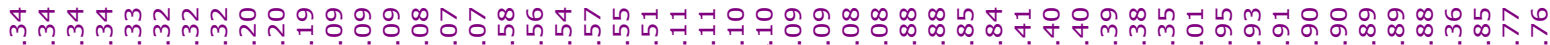

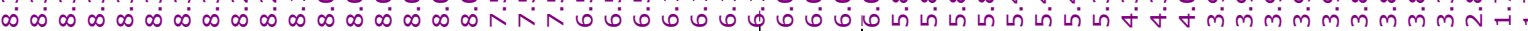
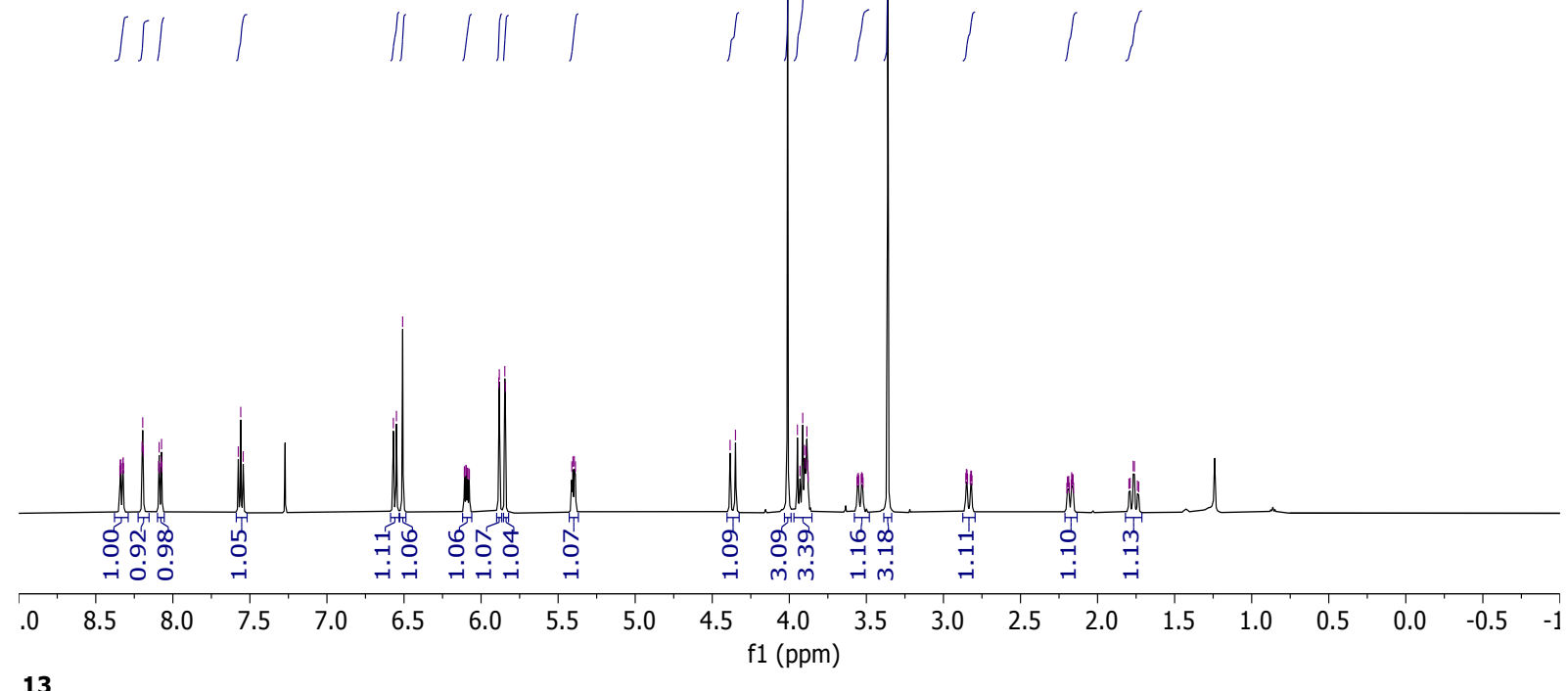

13

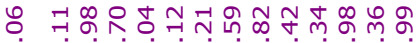

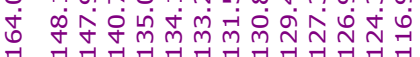

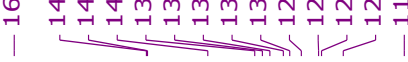

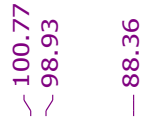

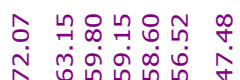

$\stackrel{\substack{i \\ \infty}}{i}$

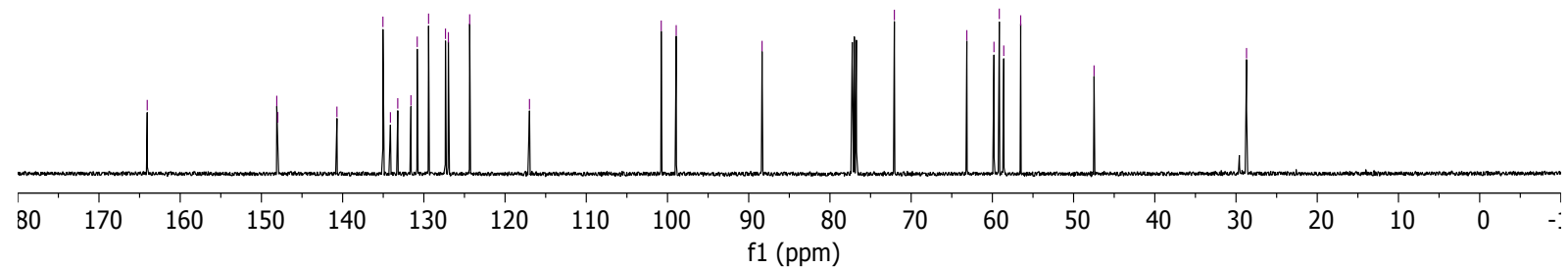


Figure S13. MS and NMR spectra of 11-O-(4-nitrobenzoyl)ambelline (14)

481.15
20171116

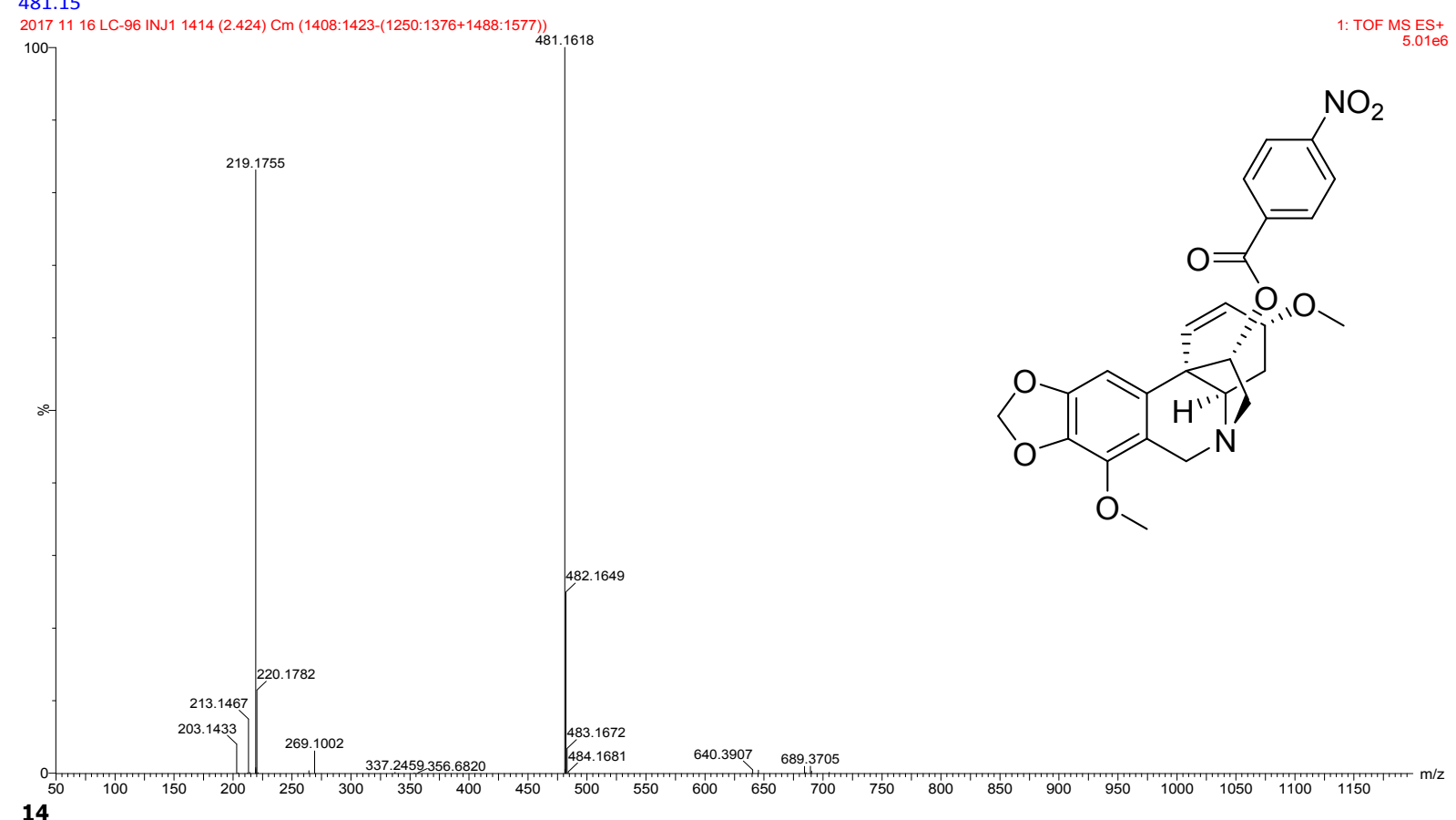
14

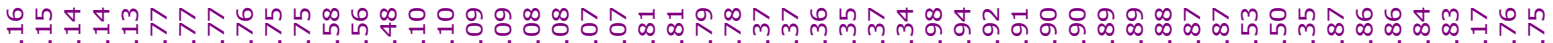

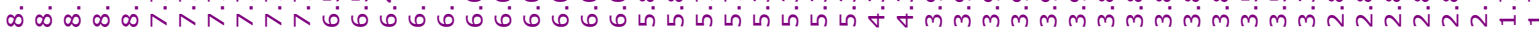<smiles>C1CCCCC1</smiles><smiles>C1=CCCCC1</smiles>
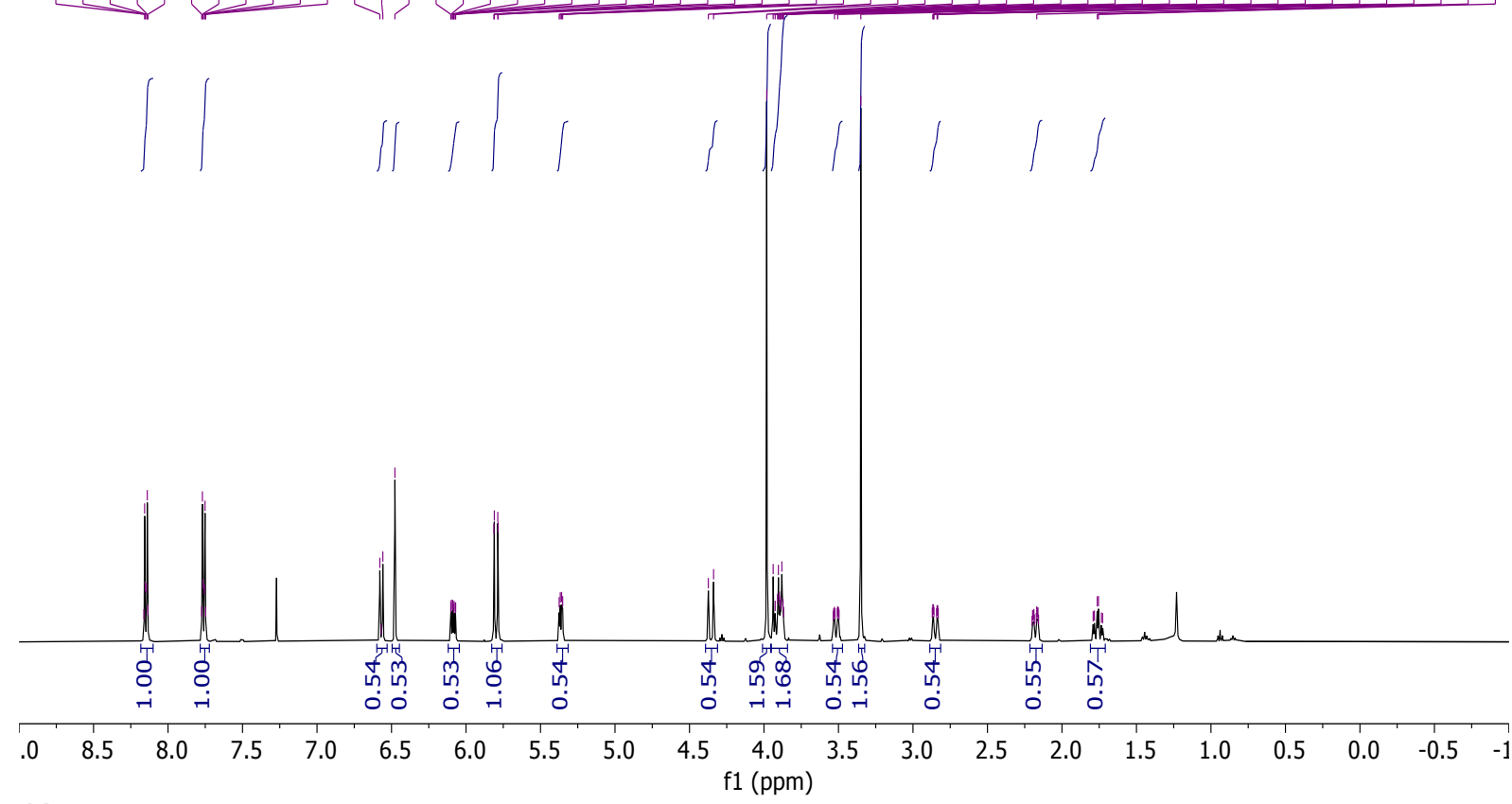

14

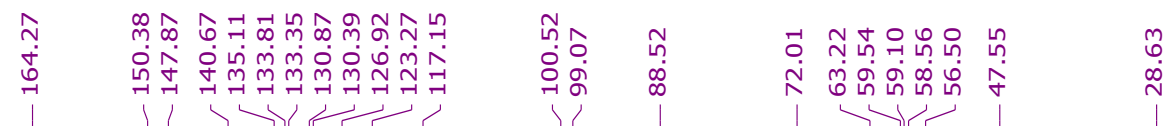

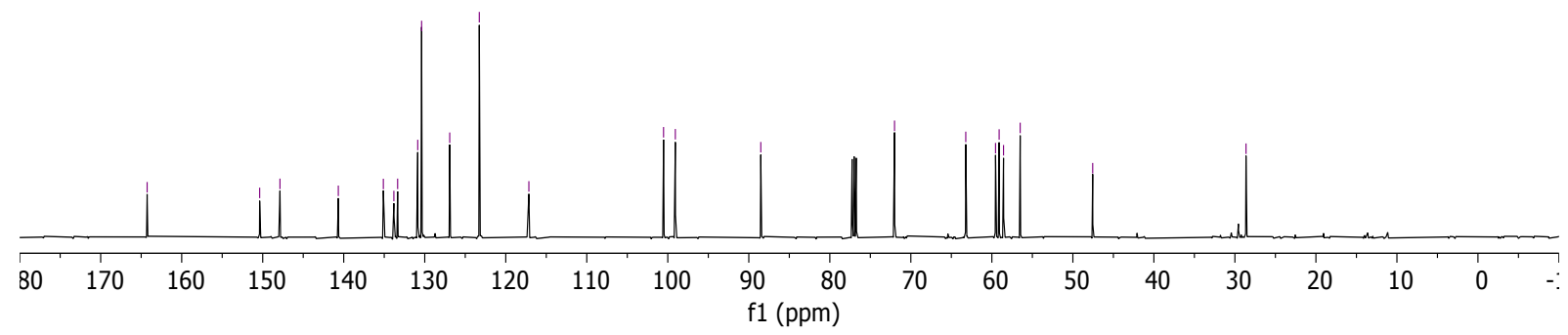


Figure S14. MS and NMR spectra of 11-O-(2-naphthoyl)ambelline (15)

486.1911
20190917 LC 174 A 1620 (2.791) Cm (1602:1634-(1438:1538+1713:1868))

100

486.1917
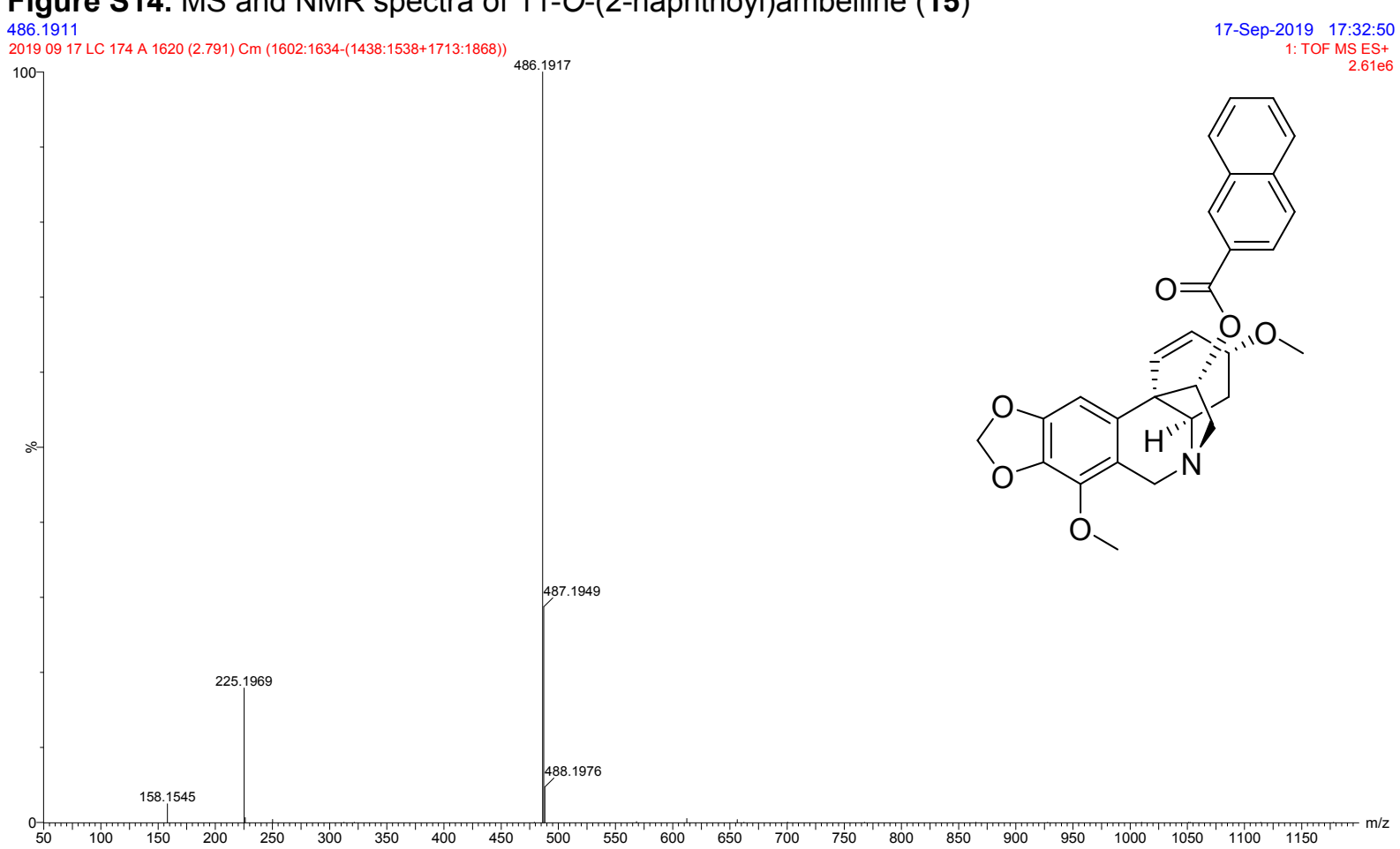

15

구

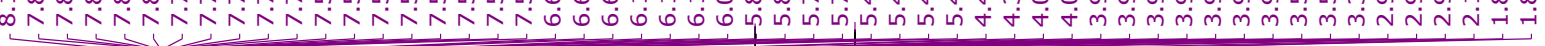

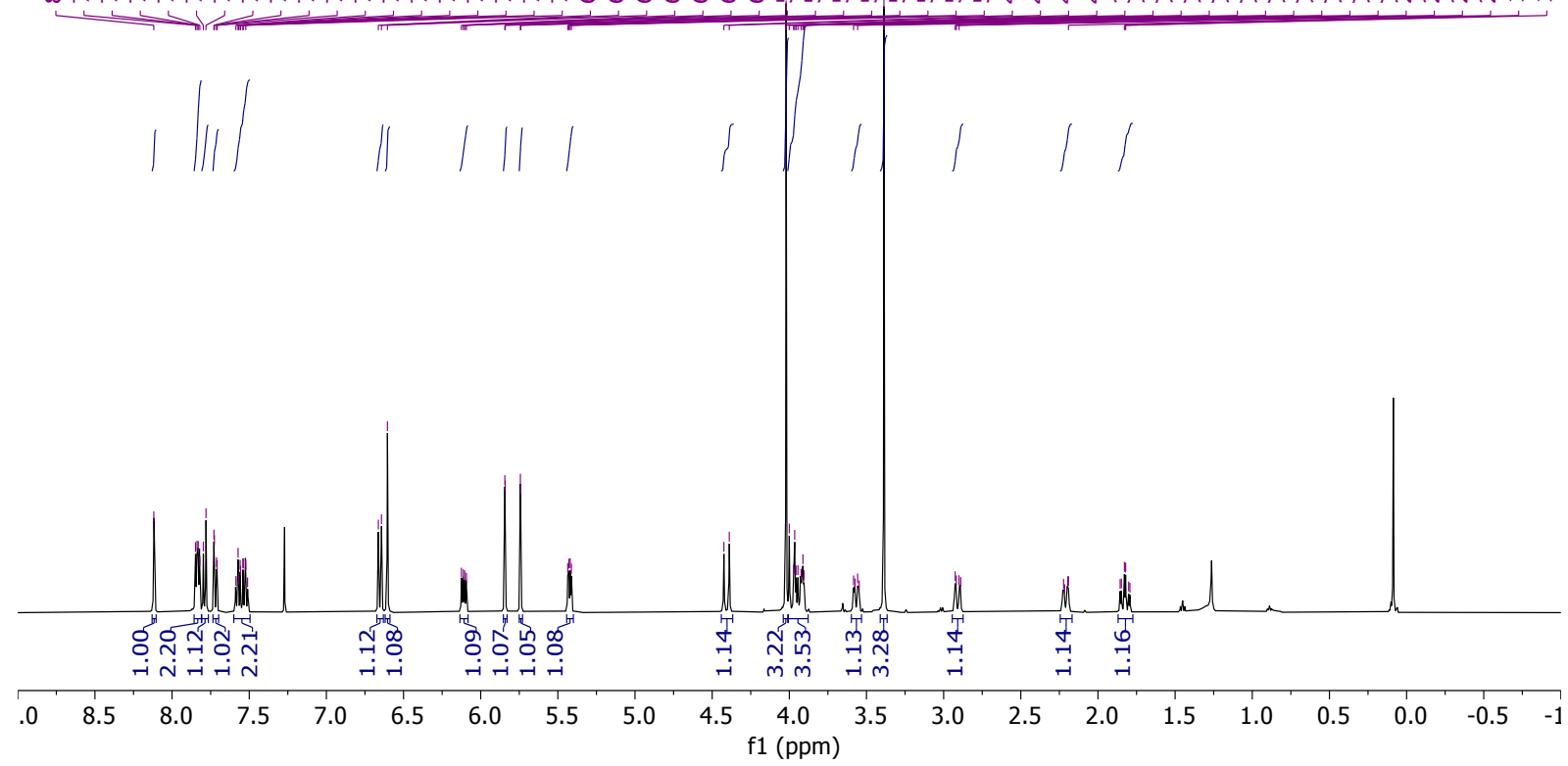

15

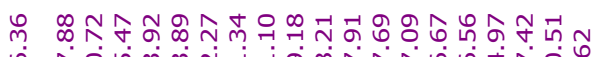

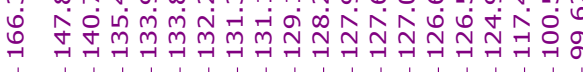

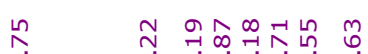

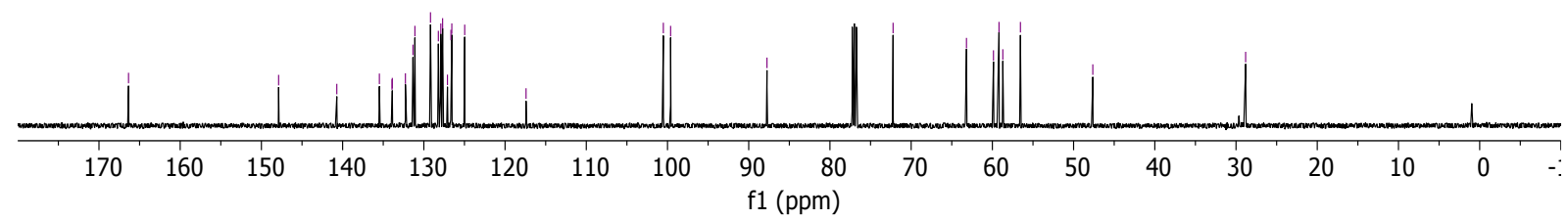


Figure S15. MS and NMR spectra of 11-O-(1-naphthoyl)ambelline (16)

\subsection{1}

20190917 LC 170 A 1581 (2.728) Cm (1573:1607-(1294:1466+1676:1878))

100

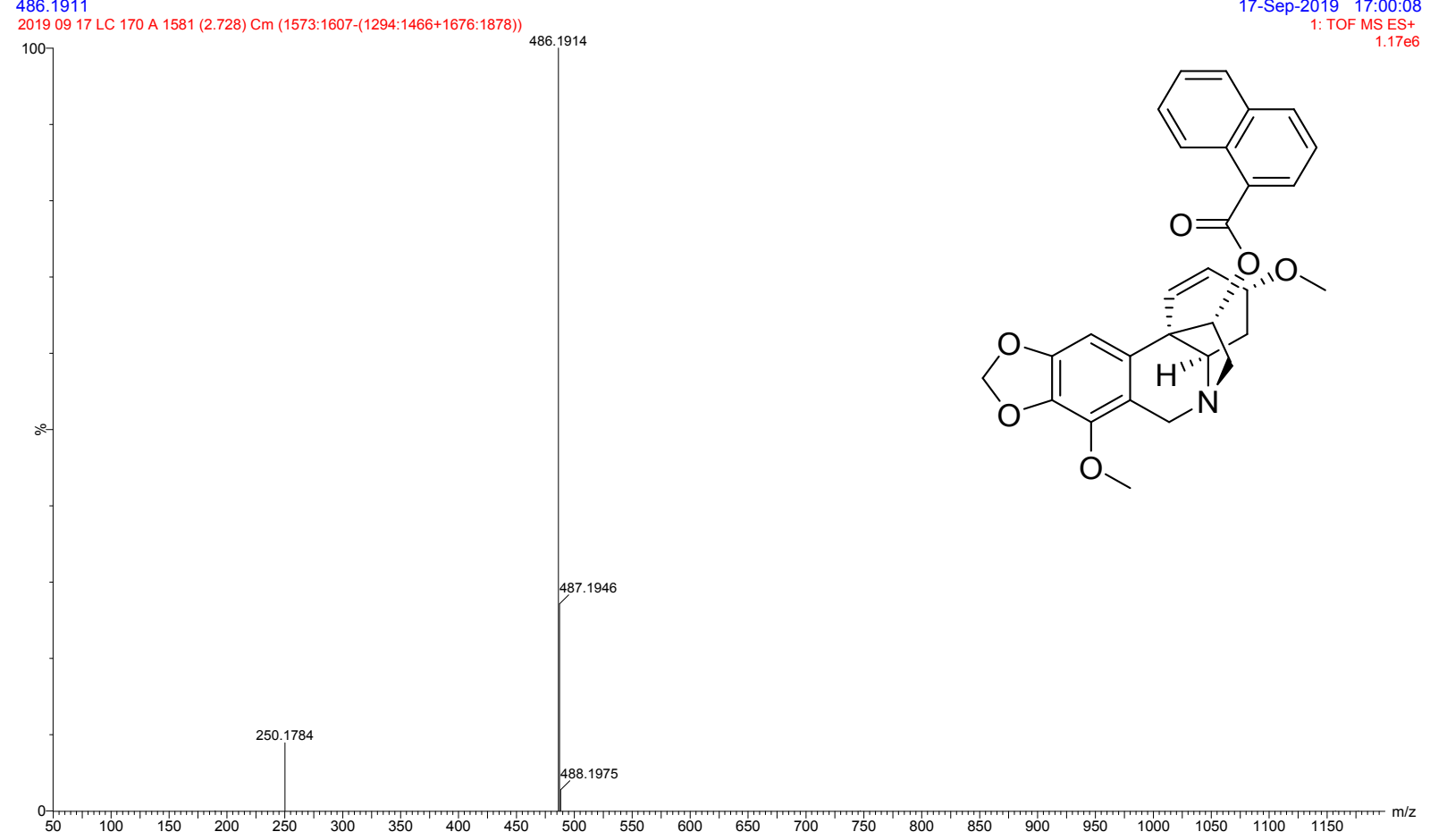

-Sep-2019 17:00:08 1: TOF MS ES+

16

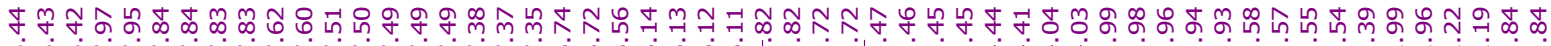

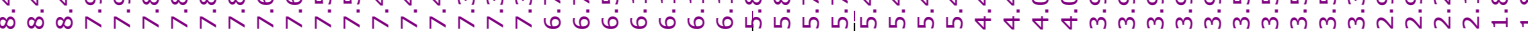
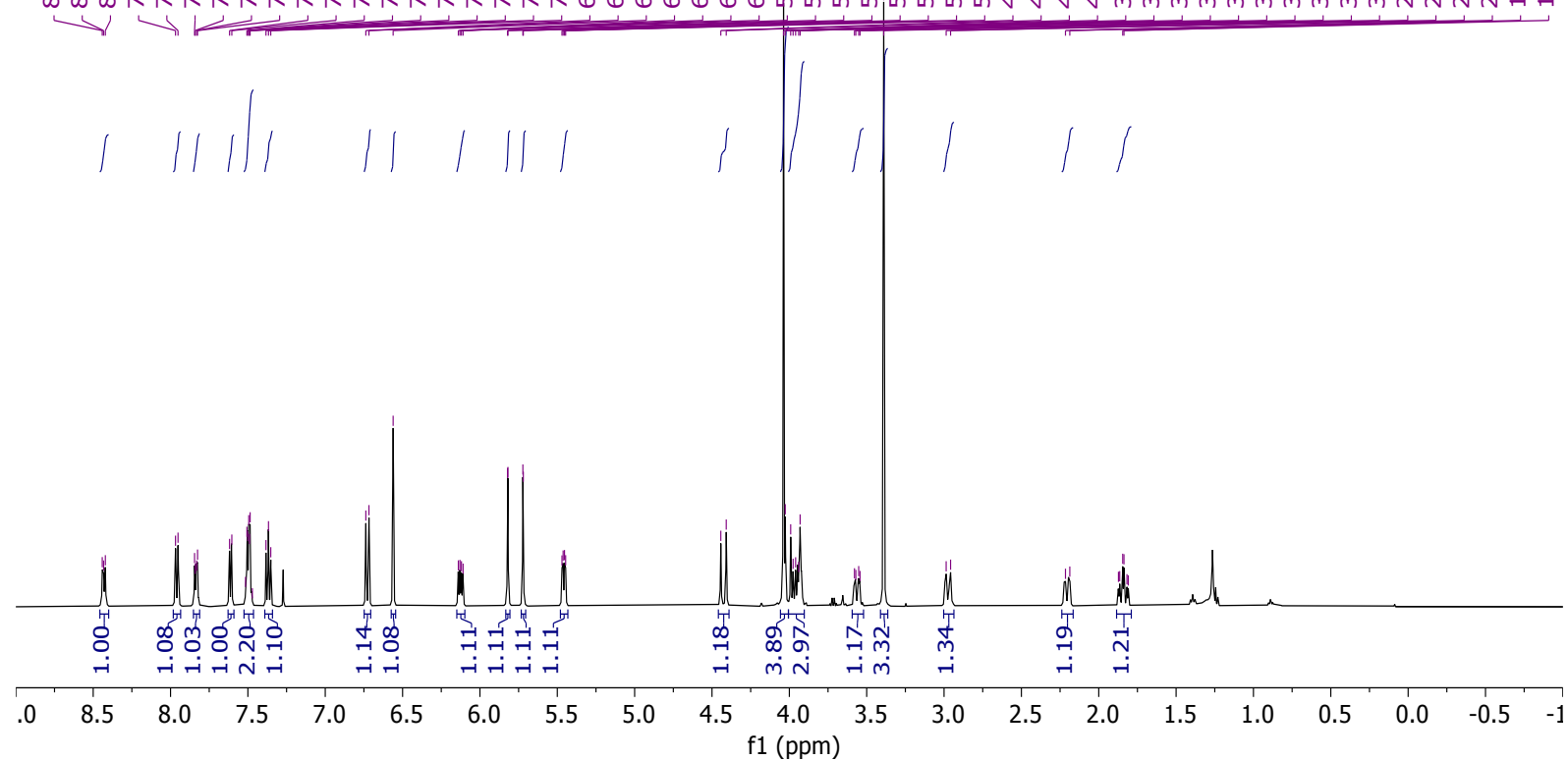

16

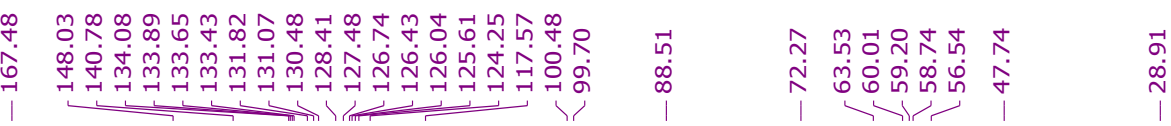

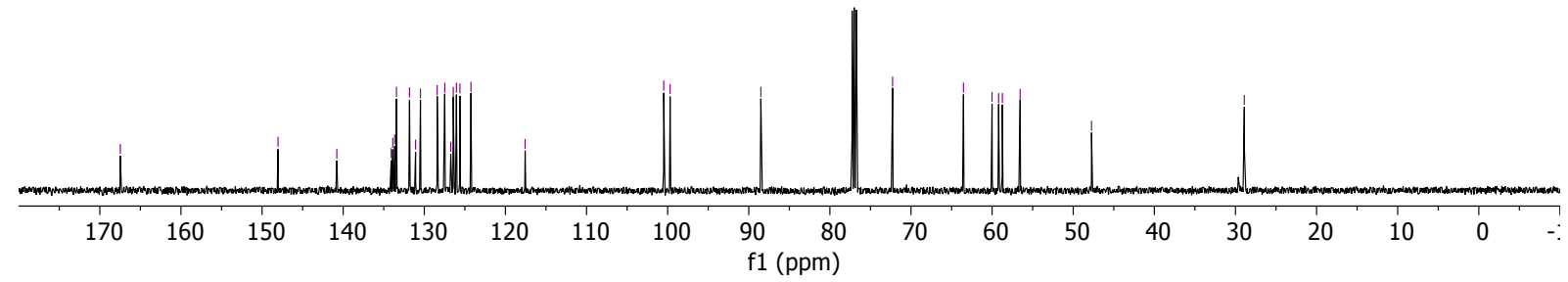


Figure S16. MS and NMR spectra of 11-O-(2-furoyl)ambelline (17)

426.1547

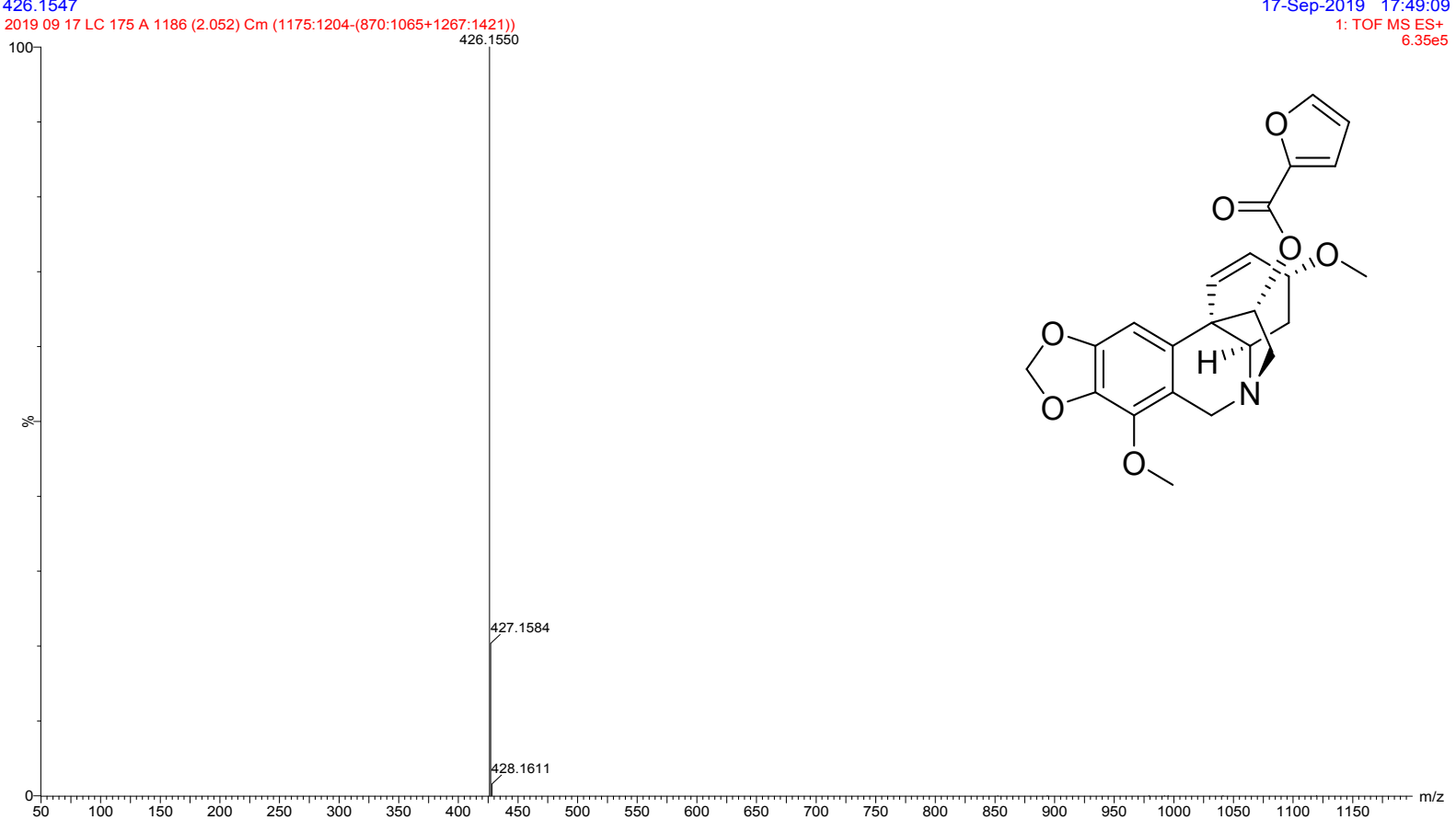

17

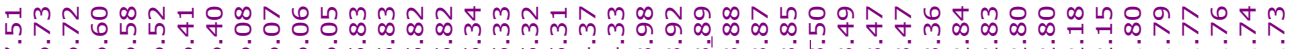

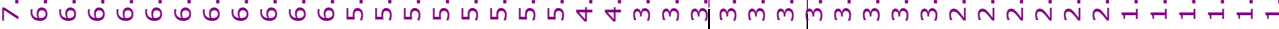

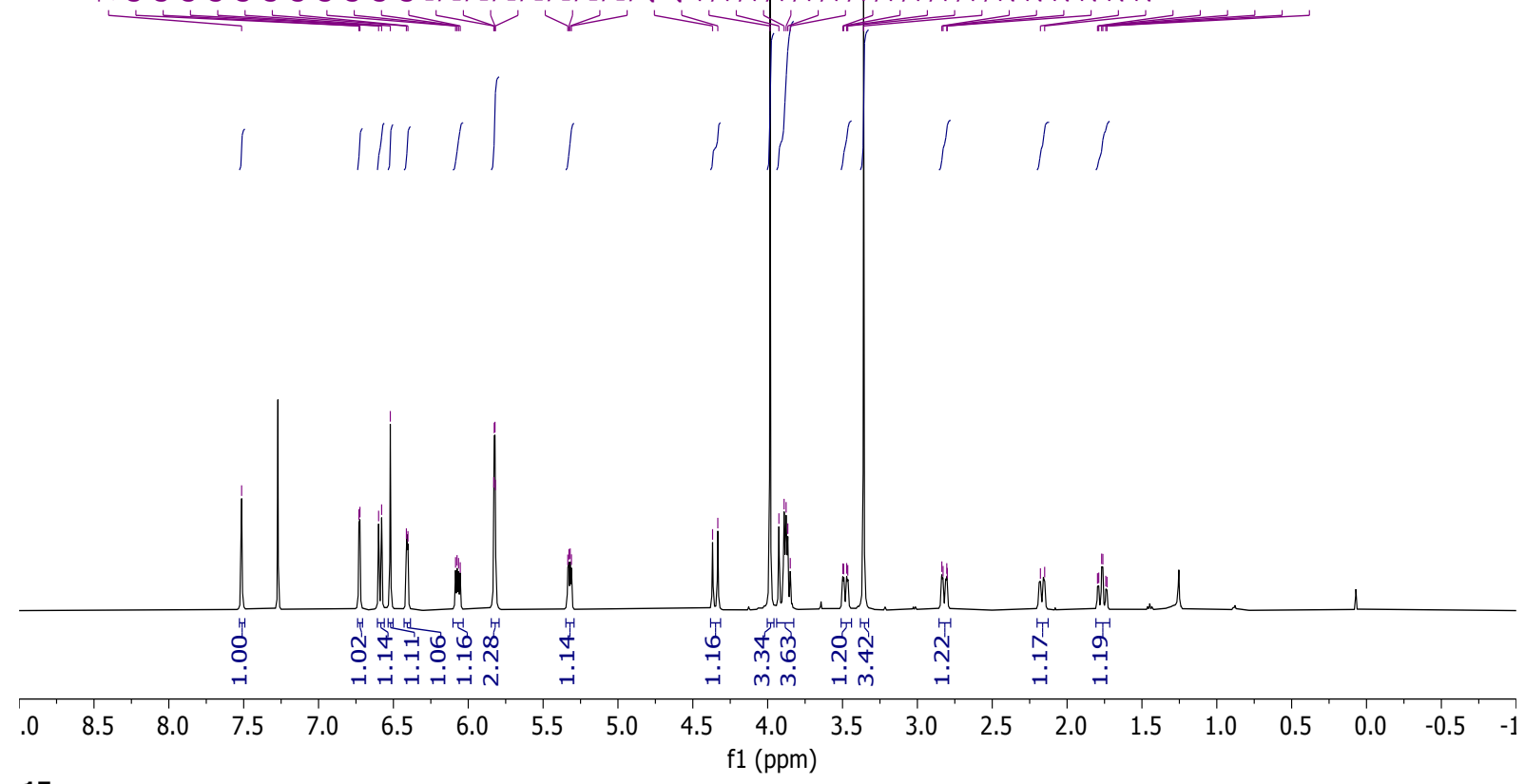

17

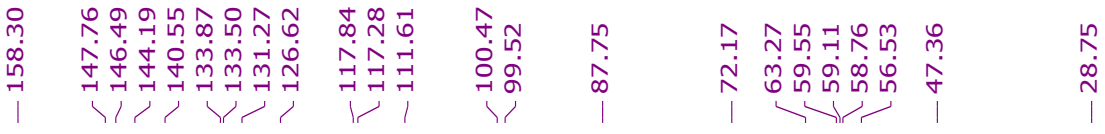

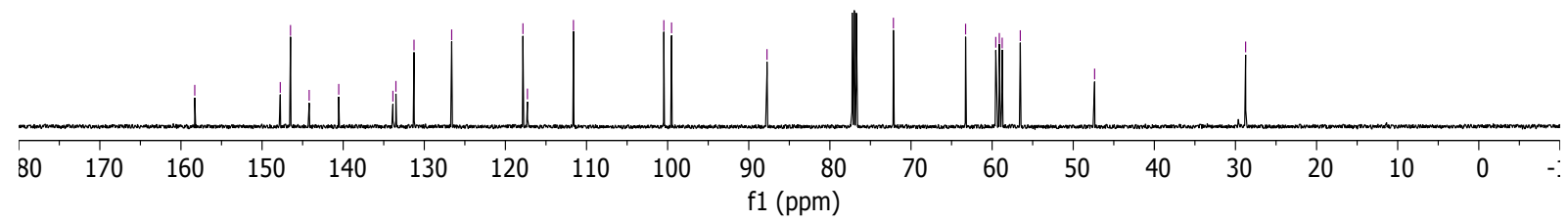


Figure S17. MS and NMR spectra of 11-O-(3-furoyl)ambelline (18)

426.1547
20190917 LC 171 A 1186 (2.051) Cm (1179:1206-(868:1072+1285:1469))
1006

17-Sep-2019 17:16:25

20190917 LC 171 A $1186(2.051)$ Cm (1179:1206-(868:1072+1285:1469))

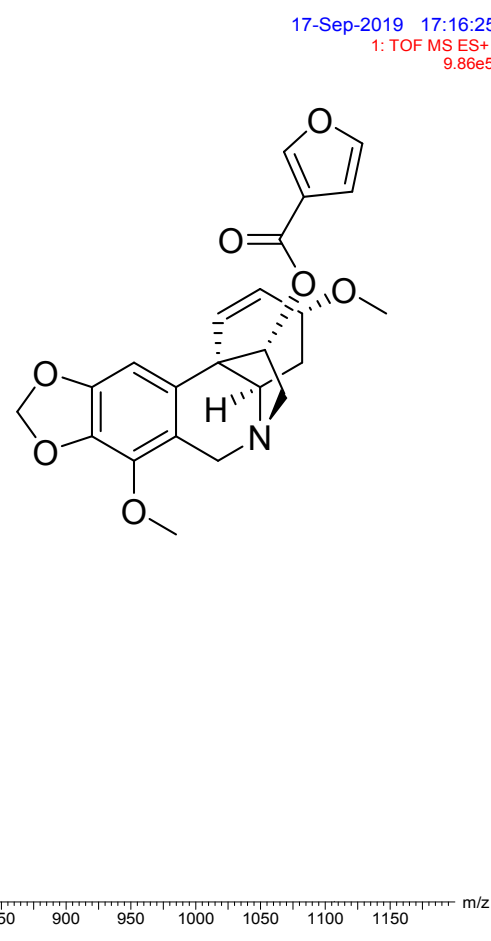

18

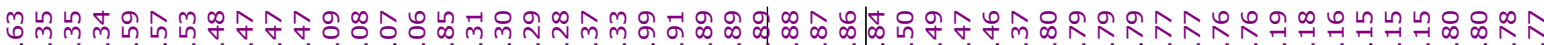
ベベベベம ம் ம் ம் ம்

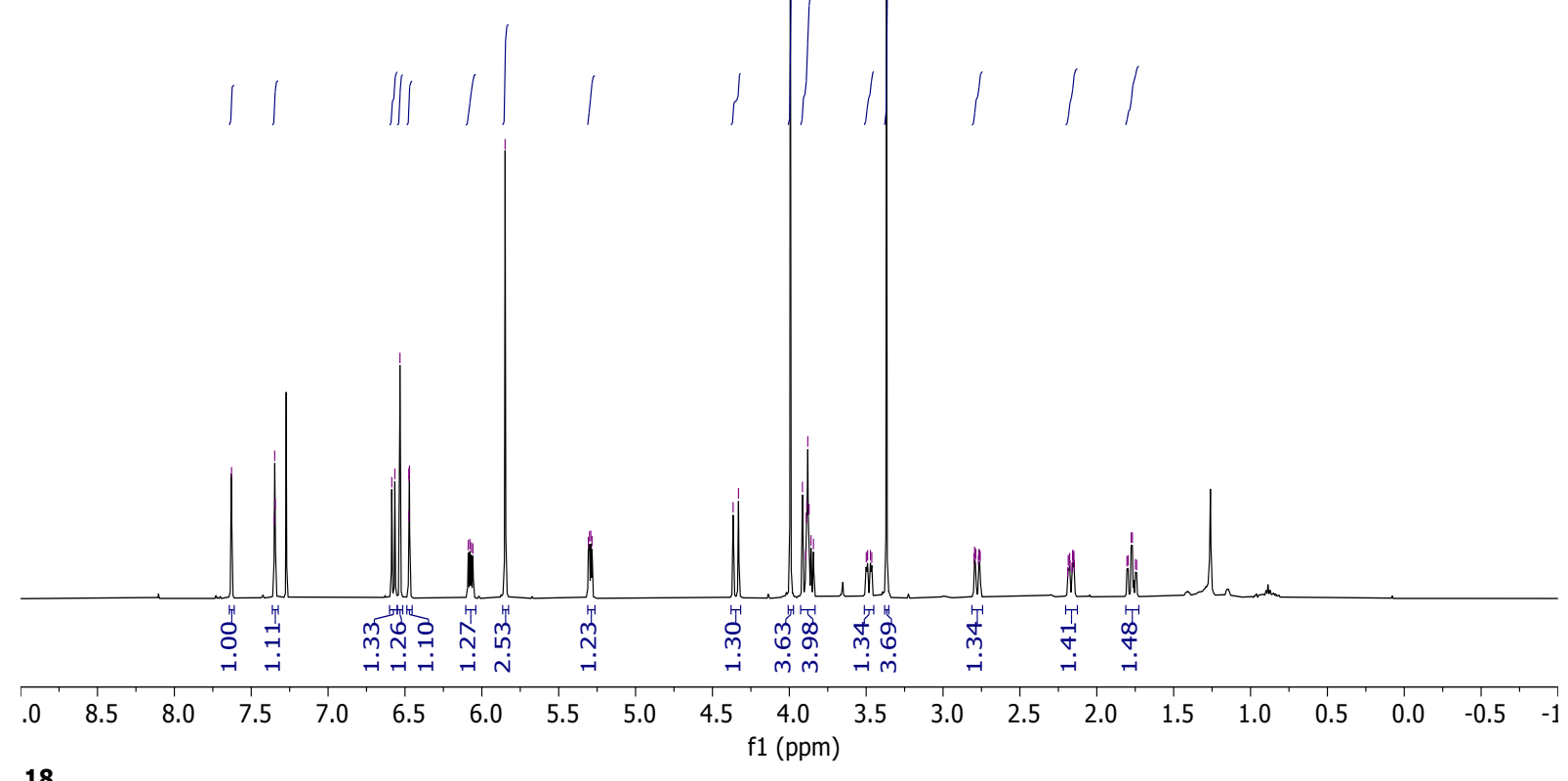

18

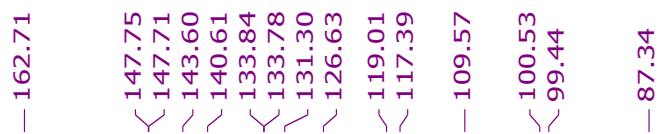

กับ

N

$\stackrel{\infty}{\infty}$

$r$ in

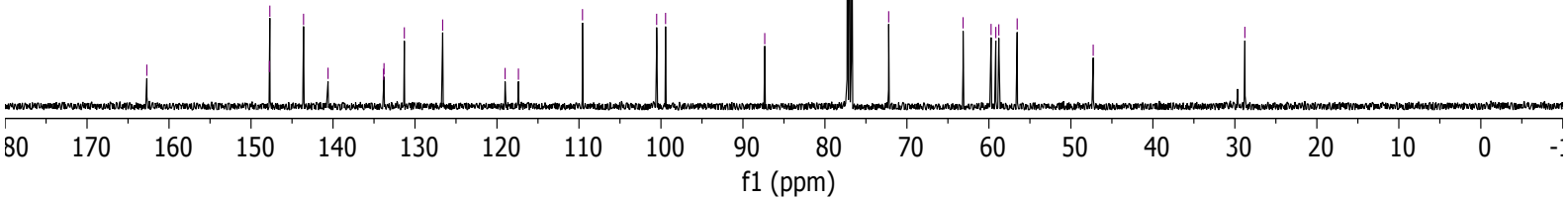


Figure S18. MS and NMR spectra of 11-O-(3,4-dimethoxybenzoyl)ambelline (19)

496.1966
20190917

(2.241) Cm (1291:1317-(1100:1242+1358:1409))

100

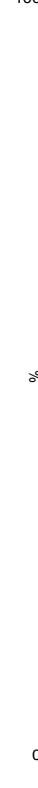

19

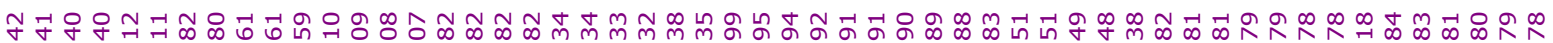
$\hat{\wedge} \wedge \wedge \wedge \wedge \dot{0} 0$ ம

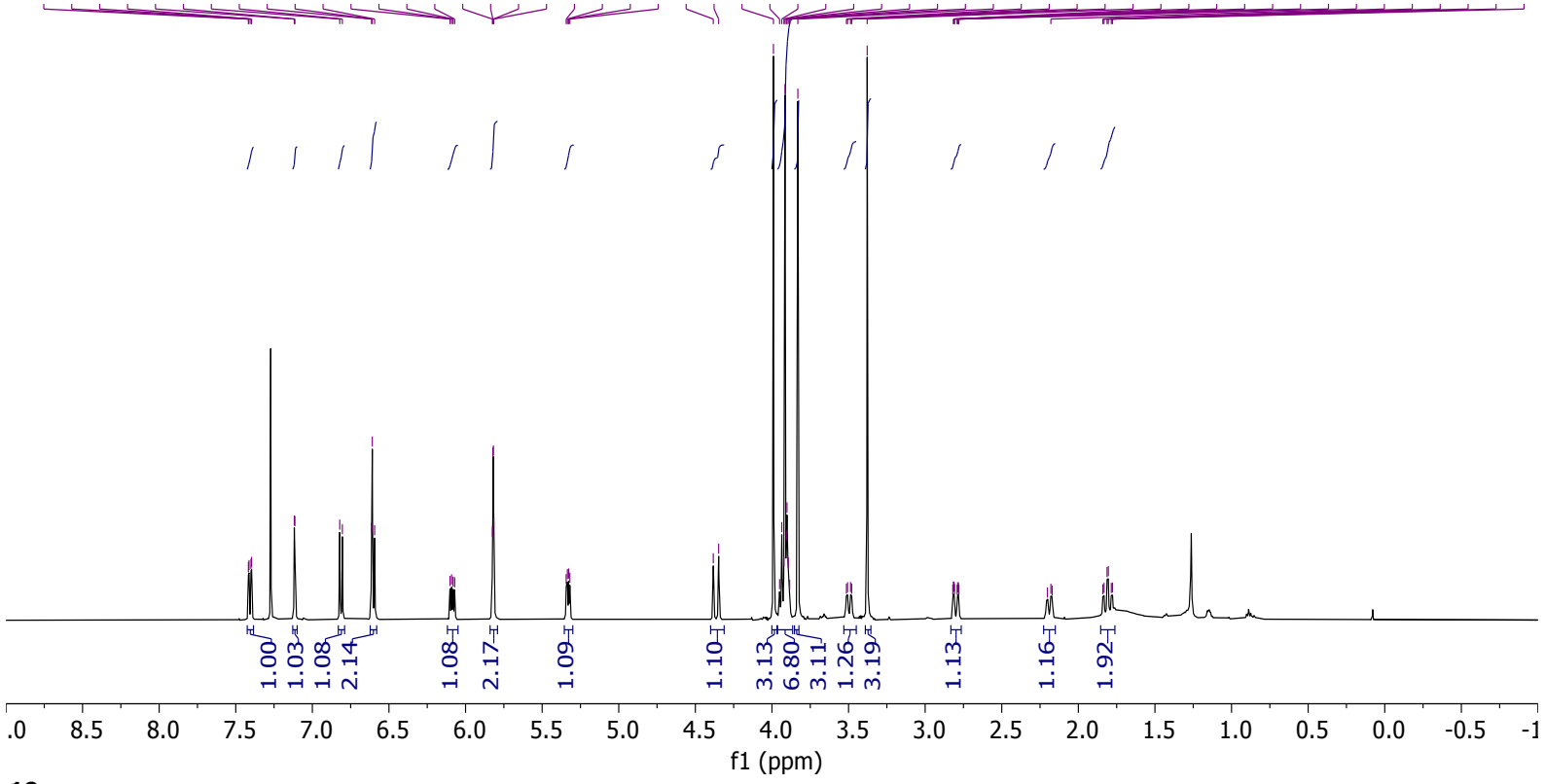

19

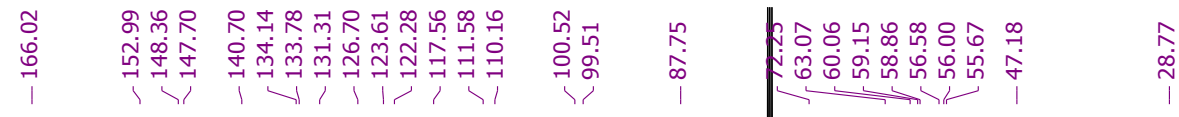

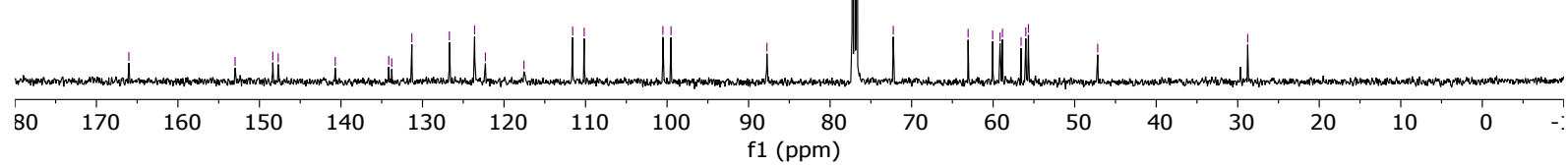


Figure S19. MS and NMR spectra of 11-O-(3,5-diethoxybenzoyl)ambelline (20)

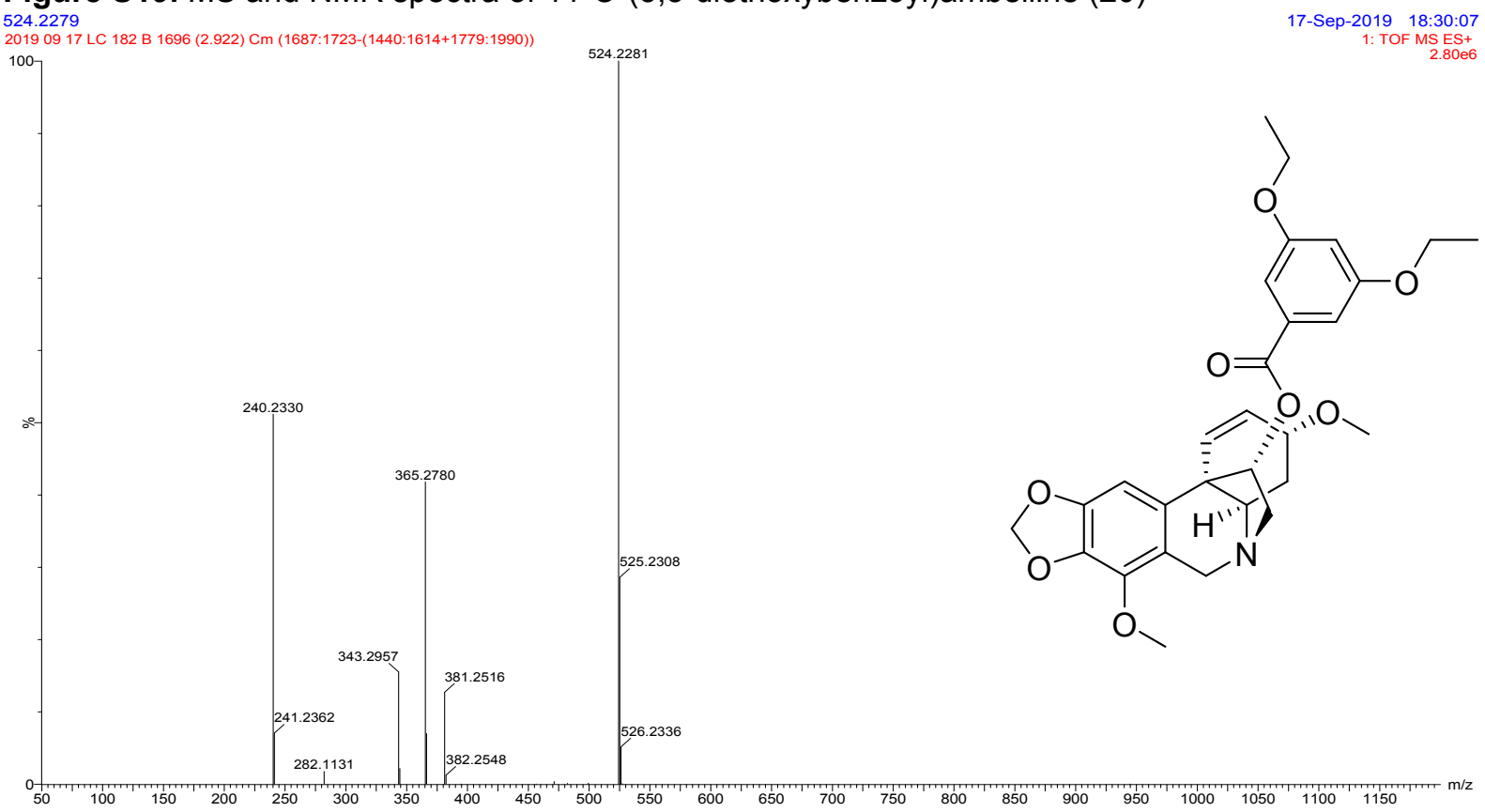

20

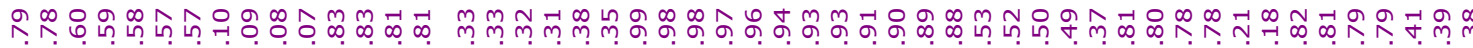
ம

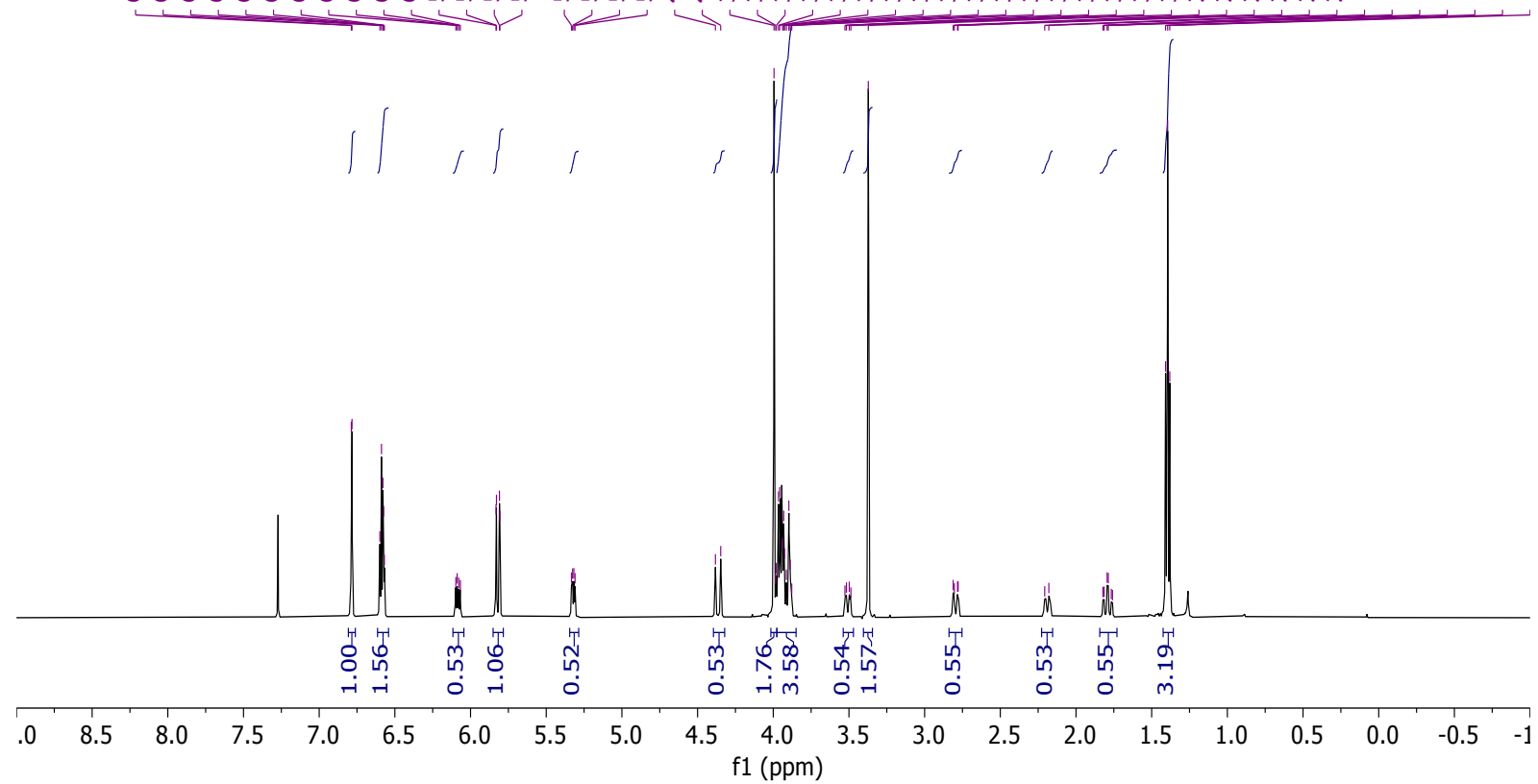

20
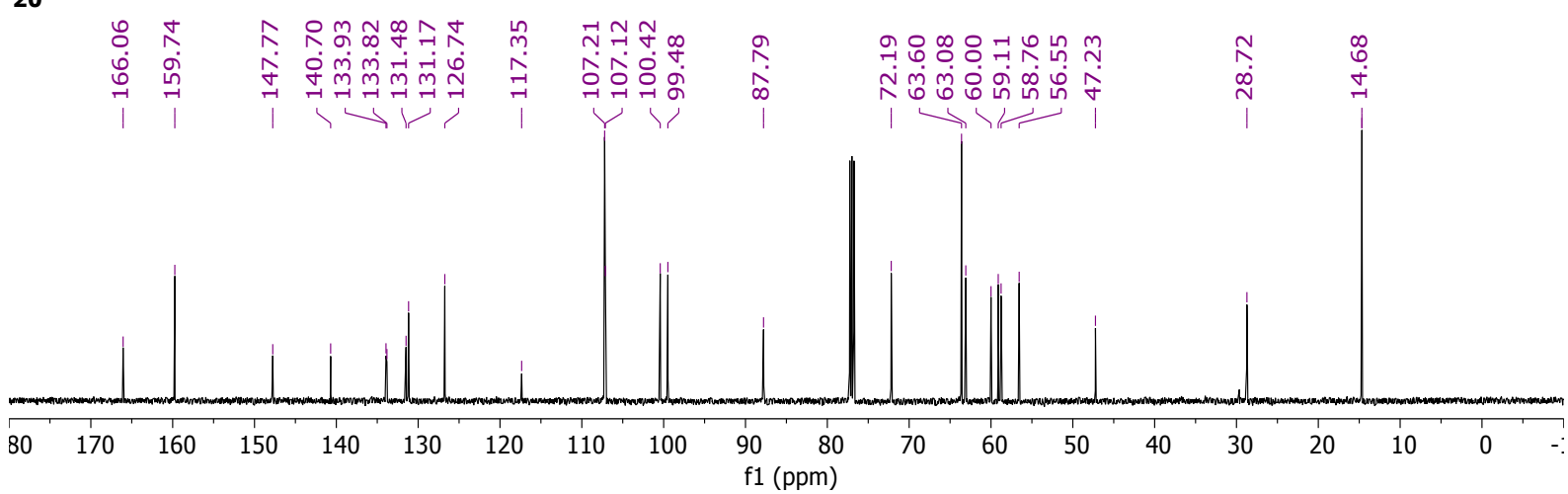
Table S1. Permeability $\left(P e 10^{-6} \mathrm{~cm} \mathrm{~s}^{-1}\right)$ in the PAMPA-BBB assay for 10 commercial drugs (used in the experiment validation) and compounds 7, 9 and $\mathbf{1 1}$ with their predictive penetration in the CNS. ${ }^{\text {a }}$

\begin{tabular}{|c|c|c|c|c|}
\hline Compound & Bibl. ${ }^{b}$ & $P e\left(10^{-6} \mathrm{cn}\right.$ & $-1)^{c}$ & Prediction \\
\hline Atenolol & 0,8 & $0,3 \pm$ & 0,3 & CNS- \\
\hline Caffeine & 1,3 & $0,1 \pm$ & 0,1 & CNS- \\
\hline Desipramine & 12 & $7,7 \pm$ & 0,4 & CNS+ \\
\hline Enoxacin & 0,9 & $0,6 \pm$ & 0,5 & CNS- \\
\hline Hydrocortisone & 1,9 & $0,8 \pm$ & 0,2 & CNS- \\
\hline Ofloxacin & 0,8 & $0,3 \pm$ & 0,1 & CNS- \\
\hline Piroxicam & 2,5 & $0,9 \pm$ & 0,2 & CNS- \\
\hline Promazine & 8,8 & $8,6 \pm$ & 0,5 & CNS+ \\
\hline Testosterone & 17 & $13,6 \pm$ & 0,3 & CNS+ \\
\hline Verapamil & 16 & $15,5 \pm$ & 0,8 & CNS+ \\
\hline
\end{tabular}

